\title{
Sensor and Sensor Fusion Technology in Autonomous Vehicles: A Review
}

\author{
De Jong Yeong ${ }^{1,2,3, * \mathbb{C}}$, Gustavo Velasco-Hernandez ${ }^{1,2,3} \mathbb{C}$, John Barry ${ }^{1,2,3}$ and Joseph Walsh ${ }^{1,2,3}$ \\ 1 IMaR Research Centre, Munster Technological University, V92 CX88 Tralee, Ireland; \\ gustavo.velascohernandez@staff.ittralee.ie (G.V.-H.); john.barry@staff.ittralee.ie (J.B.); \\ joseph.walsh@staff.ittralee.ie (J.W.) \\ 2 School of Science Technology, Engineering and Mathematics, Munster Technological University, \\ V92 CX88 Tralee, Ireland \\ 3 Lero-Science Foundation Ireland Research Centre for Software, V92 NYD3 Limerick, Ireland \\ * Correspondence: de.jong.yeong@research.ittralee.ie
}

Citation: Yeong, D.J.;

Velasco-Hernandez, G.; Barry, J.; Walsh, J. Sensor and Sensor Fusion Technology in Autonomous Vehicles: A Review. Sensors 2021, 21, 2140. https://doi.org/10.3390/s21062140

Academic Editor: Elfed Lewis

Received: 19 February 2021

Accepted: 15 March 2021

Published: 18 March 2021

Publisher's Note: MDPI stays neutral with regard to jurisdictional claims in published maps and institutional affiliations.

Copyright: (C) 2021 by the authors Licensee MDPI, Basel, Switzerland. This article is an open access article distributed under the terms and conditions of the Creative Commons Attribution (CC BY) license (https:// creativecommons.org/licenses/by/ $4.0 /)$.

\begin{abstract}
With the significant advancement of sensor and communication technology and the reliable application of obstacle detection techniques and algorithms, automated driving is becoming a pivotal technology that can revolutionize the future of transportation and mobility. Sensors are fundamental to the perception of vehicle surroundings in an automated driving system, and the use and performance of multiple integrated sensors can directly determine the safety and feasibility of automated driving vehicles. Sensor calibration is the foundation block of any autonomous system and its constituent sensors and must be performed correctly before sensor fusion and obstacle detection processes may be implemented. This paper evaluates the capabilities and the technical performance of sensors which are commonly employed in autonomous vehicles, primarily focusing on a large selection of vision cameras, LiDAR sensors, and radar sensors and the various conditions in which such sensors may operate in practice. We present an overview of the three primary categories of sensor calibration and review existing open-source calibration packages for multi-sensor calibration and their compatibility with numerous commercial sensors. We also summarize the three main approaches to sensor fusion and review current state-of-the-art multi-sensor fusion techniques and algorithms for object detection in autonomous driving applications. The current paper, therefore, provides an end-to-end review of the hardware and software methods required for sensor fusion object detection. We conclude by highlighting some of the challenges in the sensor fusion field and propose possible future research directions for automated driving systems.
\end{abstract}

Keywords: autonomous vehicles; self-driving cars; perception; camera; lidar; radar; sensor fusion; calibration; obstacle detection

\section{Introduction}

According to the Global Status Report published by the World Health Organization (WHO), the reported number of annual road traffic deaths reached 1.35 million in 2018, making it the world's eighth leading cause of unnatural death among people of all ages [1]. In the context of the European Union (EU), while there has been a decrease in the reported annual road fatalities, there is still more than 40,000 fatalities per annum, $90 \%$ of which were caused by human error. For this reason and to improve traffic flows, global investors have invested significantly to support the development of self-driving vehicles. Additionally, it is expected that the autonomous vehicles (AVs) will help to reduce the level of carbon emissions, and hence contribute to carbon emissions reduction targets [2].

AVs or self-driving vehicles provide the transportation capabilities of conventional vehicles but are largely capable of perceiving the environment and self-navigating with minimal or no human intervention. According to a report published by the Precedence Research, the global AV market size reached approximately 6500 units in 2019 and is 
predicted to experience a compound annual growth rate of $63.5 \%$ over the period 2020 to 2027 [3]. In 2009, Google secretly initiated its self-driving car project, currently known as Waymo (and presently a subsidiary of Google parent company Alphabet). In 2014, Waymo revealed a $100 \%$ autonomous car prototype without pedals and steering wheel [4]. To date, Waymo has achieved a significant milestone, whereby its AVs had collectively driven over 20 million miles on public roads in 25 cities in the United States of America (USA) [5]. Within the Irish context, in 2020, Jaguar Land Rover (JLR) Ireland has announced its collaboration with autonomous car hub in Shannon, Ireland, and will use $450 \mathrm{~km}$ of roads to test its next-generation AV technology [6].

In 2014, the SAE International, previously known as the Society of Automotive Engineers (SAE) introduced the J3016 “Levels of Driving Automation" standard for consumers. The J3016 standard defines the six distinct levels of driving automation, starting from SAE level 0 where the driver is in full control of the vehicle, to SAE level 5 where vehicles can control all aspects of the dynamic driving tasks without human intervention. The overview of these levels is depicted in Figure 1 and are often cited and referred to by industry in the safe design, development, testing, and deployment of highly automated vehicles (HAVs) [7]. Presently, automobile manufacturers such as Audi (Volkswagen) and Tesla adopted the SAE level 2 automation standards in developing its automation features, namely Tesla's Autopilot [8] and Audi A8's Traffic Jam Pilot [9,10]. Alphabet's Waymo, on the other hand, has since 2016 evaluated a business model based on SAE level 4 self-driving taxi services that could generate fares within a limited area in Arizona, USA [11].

\begin{tabular}{|c|c|c|}
\hline SAE Level 0 & SAE Level 1 & SAE Level 2 \\
\hline $\begin{array}{c}\text { NO } \\
\text { AUTOMATION }\end{array}$ & $\begin{array}{c}\text { DRIVER } \\
\text { ASSISTANCE }\end{array}$ & $\begin{array}{c}\text { PARTIAL } \\
\text { AUTOMATION }\end{array}$ \\
\hline $\begin{array}{l}\text { The human } \\
\text { driver performs } \\
\text { all driving } \\
\text { aspects of } \\
\text { driving tasks, } \\
\text { e.g., steering, } \\
\text { acceleration, etc. }\end{array}$ & $\begin{array}{l}\text { The vehicle } \\
\text { features a single } \\
\text { automated } \\
\text { system for driver } \\
\text { assistance, such } \\
\text { as steering or } \\
\text { acceleration/dece } \\
\text { leration and with } \\
\text { the anticipation } \\
\text { that the human } \\
\text { driver performs } \\
\text { all remaining } \\
\text { aspects of the } \\
\text { driving tasks. }\end{array}$ & $\begin{array}{l}\text { ADAS. The } \\
\text { vehicle can } \\
\text { perform steering } \\
\text { and } \\
\text { acceleration/dece } \\
\text { leration. } \\
\text { However, the } \\
\text { human driver is } \\
\text { required to } \\
\text { monitor the } \\
\text { driving } \\
\text { environment and } \\
\text { can take control } \\
\text { at any time. }\end{array}$ \\
\hline
\end{tabular}

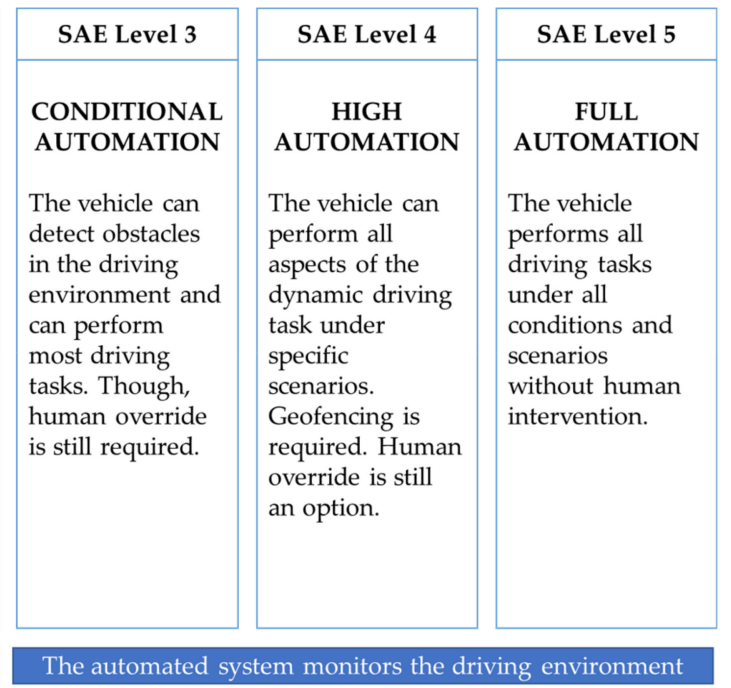

Figure 1. An overview of the six distinct levels of driving automation that were described in the Society of Automotive Engineers (SAE) J3016 standard. Readers interested in the comprehensive descriptions of each level are advised to refer to SAE International. Figure redrawn and modified based on depictions in [7].

Most autonomous driving (AD) systems share many common challenges and limitations in real-world situations, e.g., safe driving and navigating in harsh weather conditions, and safe interactions with pedestrians and other vehicles. Harsh weather conditions, such as glare, snow, mist, rain, haze, and fog, can significantly impact the performance of the perception-based sensors for perception and navigation. Besides, the challenges for $\mathrm{AD}$ in adverse weather are faced in other constrained AD scenarios like agriculture and logistics. For on-road AVs, the complexity of these challenges increases because of the unexpected conditions and behaviors from other vehicles. For example, placing a yield sign in an intersection can change the behavior of the approaching vehicles. Hence, a comprehensive prediction module in AVs is critical to identify all position future motions to reduce collision hazards $[12,13]$. Although AD systems share many common challenges in real-world situations, they are differed noticeably in several aspects. For instance, un- 
manned tractors in agriculture farm navigates between crop rows in a fixed environment, while on-road vehicles must navigate through complex dynamic environment, such as crowds and traffics [14].

While AV systems may vary slightly from one to another, all are complex systems that consists of many subcomponents. In [15], the architecture of an AD system is introduced from a technical perspective, which incorporates the hardware and software components of the AD system, and from a functional perspective, which describes the processing blocks required within the $\mathrm{AV}$, from data collection to the control of the vehicle. The hardware and software are the two primary layers from the technical perspective, and each layer includes various subcomponents that represent different aspects of the overall system. Some of the subcomponents serve as a backbone within its layer for communications between the hardware and software layers. In contrast, from the functional perspective, AV systems are composed of four primary functional blocks: perception, planning and decision, motion and vehicle control, and system supervision. These functional blocks are defined based on the processing stages and the flow of information from data collection to the control of the vehicle. The description of the technical and functional perspective of the architecture of an $\mathrm{AV}$ is represented in Figure 2. The detailed discussion of the AV architectures is beyond the scope of this paper (see [15] for a more detailed overview).

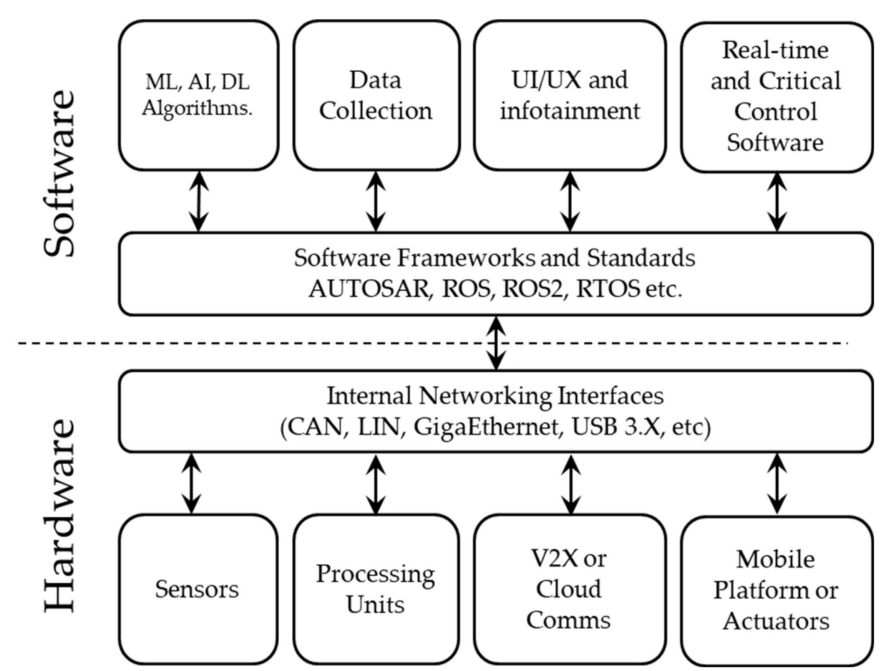

(a)

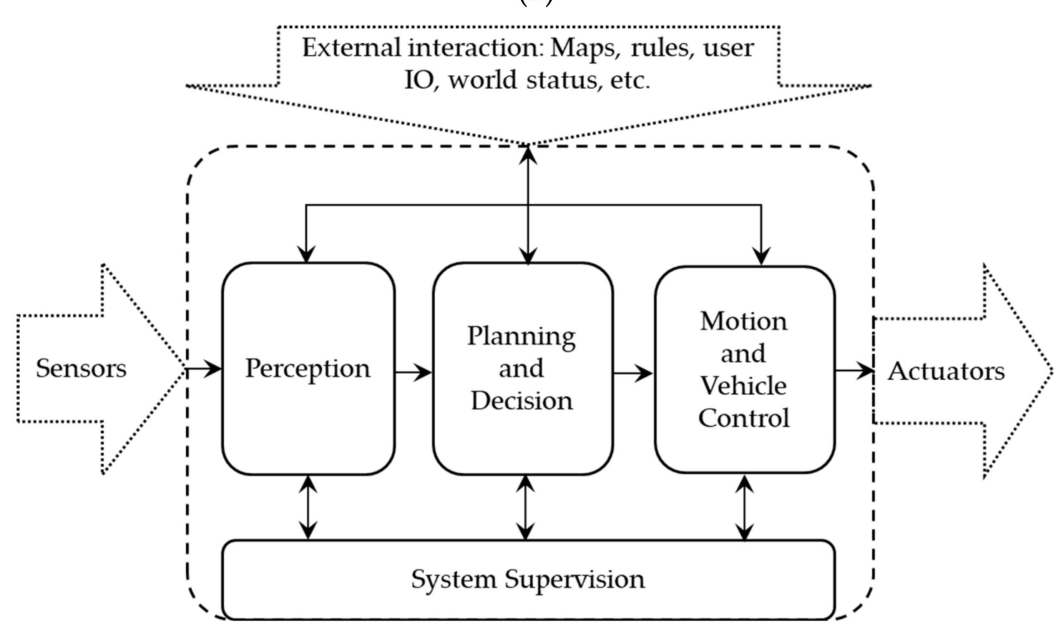

(b)

Figure 2. Architecture of an autonomous driving (AD) system from, (a) a technical perspective that describes the primary hardware and software components and their implementations; (b) a functional perspective that describes the four main functional blocks and the flow of information based on [15]. 
The sensing capabilities of an AV employing a diverse set of sensors is an essential element in the overall AD system; the cooperation and performance of these sensors can directly determine the viability and safety of an AV [16]. The selection of an appropriate array of sensors and their optimal configurations, which will, in essence be used to imitate the human ability to perceive and formulate a reliable picture of the environment, is one of the primary considerations in any AD system.

It is always essential to consider the advantages, disadvantages, and limitations of the selected group of sensors, i.e., smart sensors and non-smart sensors. The definition of "smart sensor" has evolved over the past decades along with the emergence of the Internet of Things (IoT), a system of interrelated, internet-connected objects (devices) that can collect and transfer data over the wireless network without human intervention. In the IoT context, a smart sensor is a device that can condition the input signals, process, and interpret the data, and make decisions without a separate computer [17]. In addition, in the AV context, range sensors for environment perception, e.g., cameras, LiDARs, and radars, may be considered "smart" when the sensors provide for example, target tracking, event descriptions, and other information, as part of their output. In contrast, a "non-smart" sensor is a device that only conditions the sensor raw data or waveforms and transfers the data for remote processing. It requires external computing resources to process and interpret the data to provide additional information about the environment. Ultimately, a sensor is only considered "smart" when the computer resources is an integral part of the physical sensor design [18]. Invariably, the overall performance of an AV system is greatly enhanced with multiple sensors of different types (smart/non-smart) and modalities (visual, infrared and radio waves) operating at different range and bandwidth (data rate) and with the data of each being incorporated to produce a fused output [17-19]. Multisensor fusion is effectively now a requisite process in all $\mathrm{AD}$ systems to overcome the shortcomings of individual sensor types, improving the efficiency and reliability of the overall AD system.

Several reviews have been published recently on the topic of multi-sensor fusion, some of them describing the architectural structure and sensor technologies in AVs $[15,20,21]$, or focusing on the processing stages like sensor calibration, state estimation, object and tracking [22-24], or detailing techniques used for multi-sensor fusion, like deep learningbased approaches $[19,25,26]$. Table 1 below summarizes some of the recent studies in sensor and sensor fusion technologies in AD systems.

Table 1. Summary of recent studies on sensor and sensor fusion technologies.

\begin{tabular}{|c|c|}
\hline Reference & Summary \\
\hline Velasco-Hernandez et al. [15] & $\begin{array}{l}\text { An overview of the AD architectures-technical and functional architectures depending on } \\
\text { the domain of their definition. Further, the authors highlight the perception stage of } \\
\text { self-driving solutions as a component, detailing the sensing component and sensor fusion } \\
\text { techniques to perform localization, mapping, and obstacle detection. }\end{array}$ \\
\hline Fayyad et al. [19] & $\begin{array}{l}\text { An overview of the state-of-the-art deep learning sensor fusion techniques and algorithms } \\
\text { for perception, localization, and mapping. }\end{array}$ \\
\hline Campbell et al. [20] & $\begin{array}{c}\text { A summary of sensor technologies, including their strengths and weaknesses, that were } \\
\text { commonly used to develop an autonomous vehicle. Moreover, the authors examined some } \\
\text { of the sensor fusion techniques that can be employed in both indoor and outdoor } \\
\text { environments, and algorithms for obstacle detection, navigation, and } \\
\text { environment modelling. }\end{array}$ \\
\hline Wang et al. [21] & $\begin{array}{c}\text { A discussion of sensor technology and their performance in various conditions. The authors } \\
\text { surveyed and presented a detailed summary of the multi-sensor fusion strategies in recent } \\
\text { studies and techniques to establish motion model and data association in } \\
\text { multi-target tracking. }\end{array}$ \\
\hline
\end{tabular}


Table 1. Cont.

\begin{tabular}{|c|c|}
\hline Reference & Summary \\
\hline Yeong et al. [22] & $\begin{array}{l}\text { A summary of advantages and disadvantages of perception-based sensors and the } \\
\text { architecture of multi-sensor setup for obstacle detection in industrial environments. } \\
\text { Moreover, the authors highlighted some of the challenges to temporal synchronize multiple } \\
\text { data streams in AD applications. }\end{array}$ \\
\hline Jusoh, S. \& Almajali, S. [23] & $\begin{array}{l}\text { A discussion of the current state-of-the-art multi-sensor fusion techniques and approaches } \\
\text { for various applications such as obstacle detection, localization, and mapping, in three } \\
\text { major domains, namely robotics, military, and healthcare. }\end{array}$ \\
\hline Castanedo, F. [24] & $\begin{array}{l}\text { A discussion of the classification of data fusion techniques based on several criteria and } \\
\text { providing a comprehensive overview of the most employed methods and algorithms for } \\
\text { data association, state estimation, and decision fusion tasks. }\end{array}$ \\
\hline Kuutti et al. [25] & $\begin{array}{c}\text { An overview of deep learning approaches and methods for autonomous vehicle control, } \\
\text { and the challenges to deep learning-based vehicle control. The authors considered these } \\
\text { approaches for three categories of tasks: lateral (steering), longitudinal (acceleration and } \\
\text { braking), and simultaneous lateral and longitudinal control, and discussed the relevant } \\
\text { methods in detail. }\end{array}$ \\
\hline Hu et al. [26] & $\begin{array}{l}\text { A discussion of the perception-based sensors for intelligent ground vehicles in off-road } \\
\text { environment and a comprehensive review of the current state-of-the-art multi-sensor fusion } \\
\text { approaches. In addition, the author summarized the main considerations of on-board } \\
\text { multi-sensor configurations and reviewed the architectural structure of perception systems } \\
\text { and applications for obstacle detection in diverse environments. }\end{array}$ \\
\hline
\end{tabular}

The present review paper will extend across the three major considerations in sensor fusion for AVs: Firstly, operating principles and characteristics of sensor modalities, including a comparison of commercially available hardware; secondly, the three aspects of sensor calibration, the main open-source calibration systems and their compatibility with commercial sensors; and thirdly, on sensor fusion methods and algorithms for obstacle detection in AV environments. Section 2 provides an overview of the existing sensing modalities used in AVs, primarily focusing on cameras, LiDARs, and radars, including their advantages and disadvantages, and limitations in different environmental conditions. Section 3 discusses the necessity of sensor calibration in AVs, an overview of an existing calibration package which addresses all the main aspects required by any calibration system, followed by the current developments of sensor fusion approaches for obstacle detection and its challenges for safe and reliable environment perception. Section 4 presents a summary review and recommendations for future research in $\mathrm{AVs}$.

\section{Sensor Technology in Autonomous Vehicles}

Sensors are devices that map the detected events or changes in the surroundings to a quantitative measurement for further processing. In general, sensors are classified into two classes based on their operational principal. Proprioceptive sensors, or internal state sensors, capture the dynamical state and measures the internal values of a dynamic system, e.g., force, angular rate, wheel load, battery voltage, et cetera. Examples of the proprioceptive sensors include Inertia Measurement Units (IMU), encoders, inertial sensors (gyroscopes and magnetometers), and positioning sensors (Global Navigation Satellite System (GNSS) receivers). In contrast, the exteroceptive sensors, or external state sensors, sense and acquire information such as distance measurements or light intensity from the surroundings of the system. Cameras, Radio Detection and Ranging (Radar), Light Detection and Ranging (LiDAR), and ultrasonic sensors are examples of the exteroceptive sensors. Additionally, sensors can either be passive sensors or active sensors. Passive sensors receive energy emitting from the surroundings to produce outputs, e.g., vision cameras. Conversely, active sensors emit energy into the environment and measure the environmental "reaction" to that energy to produce outputs, such as with LiDAR and radar sensors [27-29].

In AVs, sensors are critical to the perception of the surroundings and localization of the vehicles for path planning and decision making, essential precursors for controlling the 
motion of the vehicle. AV primarily utilizes multiple vision cameras, radar sensors, LiDAR sensors, and ultrasonic sensors to perceive its environment. Additionally, other sensors, including the Global Navigation Satellite System (GNSS), IMU, and vehicle odometry sensors are used to determine the relative and absolute positions of the vehicle [30]. The relative localization of an $\mathrm{AV}$ refers to the vehicles referencing of its coordinates in relation to the surrounding landmarks, while absolute localization refers to the vehicle referencing its position in relation to a global reference frame (world) [31]. The placement of sensors for environment perception on typical AV applications, their coverage, and applications are shown in Figure 3. The reader will appreciate that in a moving vehicle, there is a more complete coverage of the vehicle's surroundings. The individual and relative positioning of multiple sensors are critical for precise and accurate object detection and therefore reliably and safely performing any subsequent actions [32]. In general, it is challenging to generate adequate information from a single independent source in $\mathrm{AD}$. This section reviews the advantages and shortcomings of the three primary sensors: cameras, LiDARs and radars, for environment perception in AV applications.

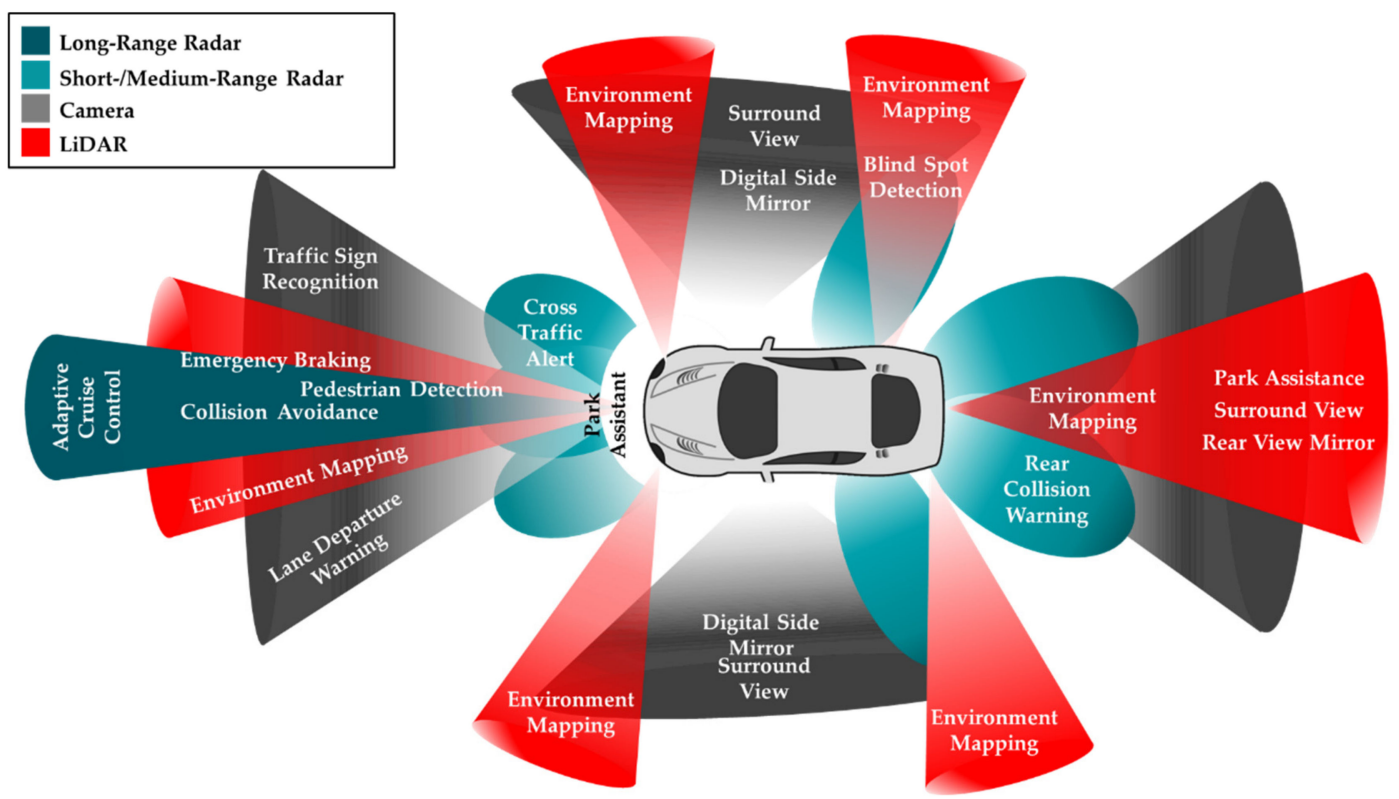

Figure 3. An example of the type and positioning of sensors in an automated vehicle to enable the vehicles perception of its surrounding. Red areas indicate the LiDAR coverage, grey areas show the camera coverage around the vehicle, blue areas display the coverage of short-range and medium-range radars, and green areas indicate the coverage of long-range radar, along with the applications the sensors enable-as depicted in [32] (redrawn).

\subsection{Camera}

Cameras are one of the most adopted technology for perceiving the surroundings. A camera works on the principle of detecting lights emitted from the surroundings on a photosensitive surface (image plane) through a camera lens (mounted in front of the sensor) to produce clear images of the surrounding [20,30]. Cameras are relatively inexpensive and with appropriate software, can detect both moving and static obstacles within their field of view and provides high-resolution images of the surroundings. These capabilities allow the perception system of the vehicle to identify road signs, traffic lights, road lane markings and barriers in the case of road traffic vehicles and a host of other articles in the case of off-road vehicles. The camera system in an AV may employ monocular cameras or binocular cameras, or a combination of both. As the name implies, the monocular camera system utilizes a single camera to create a series of images. The conventional RGB monocular cameras are fundamentally more limited than stereo cameras in that they lack native depth information, although in some applications or more advanced monocular 
cameras using the dual-pixel autofocus hardware, depth information may be calculated using complex algorithms [33-35]. As a result, two cameras are often installed side-by-side to form a binocular came-ra system in autonomous vehicles.

The stereo camera, also known as a binocular camera, imitates the perception of depth found in animals, whereby the "disparity" between the slightly different images formed in each eye is (subconsciously) employed to provide a sense of depth. Stereo cameras contain two image sensors, separated by a baseline. The term baseline refers to the distance between the two image sensors (and is generally cited in the specifications of stereo cameras), and it differs depending on the camera's model. For example, the Orbbec 3D cameras reviewed in [36] for Autonomous Intelligent Vehicles (AIV) has a baseline of $75 \mathrm{~mm}$ for both the Persee and Astra series cameras [37]. As in the case of animal vision, the disparity maps calculated from the stereo camera imagery permit the generation of depth maps using epipolar geometry and triangulation methods (detailed discussion of the disparity calculations algorithms is beyond the scope of this paper). Reference [38] uses the "stereo_image_proc" modules in Robotic Operating System (ROS), an open source, metaoperating system for robotics [39], to perform stereo vision processing before implementing SLAM (simultaneous localization and mapping) and autonomous navigation. Table 2 shows the general specifications for binocular cameras from different manufacturers.

Table 2. General specifications of stereo cameras from various manufacturers that we reviewed from our initial findings. The acronyms from left to right (in second row) are horizontal field-of-view (HFOV); vertical field-of-view (VFOV); frames per second (FPS); image resolutions in megapixels (Img Res); depth resolutions (Res); depth frames per second (FPS); and reference (Ref). The "-" symbol in table below indicates that the specifications were not mentioned in product datasheet.

\begin{tabular}{|c|c|c|c|c|c|c|c|c|c|c|c|}
\hline & \multirow[b]{2}{*}{ Model } & \multirow[b]{2}{*}{$\begin{array}{l}\text { Baseline } \\
(\mathrm{mm})\end{array}$} & \multirow[b]{2}{*}{ HFOV $\left({ }^{\circ}\right)$} & \multirow[b]{2}{*}{$\operatorname{VFOV}\left({ }^{\circ}\right)$} & \multirow[b]{2}{*}{ FPS (Hz) } & \multirow[b]{2}{*}{$\begin{array}{l}\text { Range } \\
(\mathrm{m})\end{array}$} & \multicolumn{5}{|c|}{ Depth Information } \\
\hline & & & & & & & $\begin{array}{l}\text { Img Res } \\
\text { (MP) }\end{array}$ & $\begin{array}{l}\text { Range } \\
(\mathrm{m})\end{array}$ & Res (MP) & $\begin{array}{l}\text { FPS } \\
(\mathrm{Hz})\end{array}$ & Ref \\
\hline Roboception & $\begin{array}{c}\text { RC Visard } \\
160\end{array}$ & 160 & $61 *$ & $48^{*}$ & 25 & $0.5-3$ & 1.2 & $0.5-3$ & $0.03-1.2$ & $0.8-25$ & {$[40,41]$} \\
\hline \multirow{2}{*}{$\begin{array}{l}\text { Carnegie } \\
\text { Robotics }\end{array}$} & $\begin{array}{l}\text { MultiSense }^{\mathrm{TM}} \\
\mathrm{S}^{1}{ }^{1}\end{array}$ & 70 & 80 & $49 / 80$ & $30 \max$ & - & $2 / 4$ & $0.4 \mathrm{~min}$ & $0.5-2$ & $7.5-30$ & {$[40,42,43]$} \\
\hline & $\begin{array}{l}\text { MultiSense }^{\mathrm{TM}} \\
\text { S21B }^{1}\end{array}$ & 210 & $68-115$ & $40-68$ & $30 \max$ & - & $2 / 4$ & $0.4 \mathrm{~min}$ & $0.5-2$ & $7.5-30$ & {$[40,44]$} \\
\hline Ensenso & $\begin{array}{c}\text { N35-606-16- } \\
\text { BL }\end{array}$ & 100 & 58 & 52 & 10 & $4 \max$ & 1.3 & \multicolumn{3}{|c|}{-} & {$[40,45]$} \\
\hline Framos & D435e & 55 & 86 & 57 & 30 & $0.2-10$ & 2 & $0.2 \mathrm{~min}$ & 0.9 & 30 & {$[40,46]$} \\
\hline Nerian & Karmin $^{2}$ & $50 / 100 / 250$ & 82 & 67 & 7 & - & 3 & $\begin{array}{c}0.23 / 0.45 / \\
1.14 \mathrm{~min}\end{array}$ & 2.7 & - & {$[40,47]$} \\
\hline \multirow{3}{*}{$\begin{array}{c}\text { Intel } \\
\text { RealSense }\end{array}$} & D455 & 95 & 86 & 57 & 30 & $20 \max$ & 3 & $0.4 \mathrm{~min}$ & $\leq 1$ & $\leq 90$ & \multirow{3}{*}[40,48]{} \\
\hline & D435 & 50 & 86 & 57 & 30 & $10 \max$ & 3 & $0.105 \mathrm{~min}$ & $\leq 1$ & $\leq 90$ & \\
\hline & D415 & 55 & 65 & 40 & 30 & $10 \max$ & 3 & $0.16 \mathrm{~min}$ & $\leq 1$ & $\leq 90$ & \\
\hline \multirow{2}{*}{ Flir $^{\circledR}$} & $\underset{3}{\text { Bumblebee2 }}$ & 120 & 66 & - & $48 / 20$ & - & $0.3 / 0.8$ & & \multirow[t]{2}{*}{ - } & & {$[40,49]$} \\
\hline & $\begin{array}{c}\text { Bumblebee } \\
\text { XB3 }^{3}\end{array}$ & 240 & 66 & - & 16 & - & 1.2 & & & & {$[50,51]$} \\
\hline
\end{tabular}

${ }^{1} \mathrm{HFOV}, \mathrm{VFOV}$, image resolutions, image frame rates and depth information depend on the variant of focal length (optical lens geometry). ${ }^{2}$ Specifications stated are in full resolution and monochrome, focusing on the standard $4 \mathrm{~mm}$ lens. ${ }^{3}$ Offers either $2.5 \mathrm{~mm}, 3.8 \mathrm{~mm}$ or $6 \mathrm{~mm}$ lenses (specifications focus on $3.8 \mathrm{~mm}$ lens) but product no longer being produced or offered (discontinued). ${ }^{*} \mathrm{~A} 6 \mathrm{~mm}$ lens has a HFOV of $43^{\circ}$ and a VFOV of $33^{\circ}$.

Other commonly employed cameras in AVs for perception of the surroundings include fisheye cameras [52-54]. Fisheye cameras are commonly employed in near-field sensing applications, such as parking and traffic jam assistance, and require only four cameras to provide a 360-degree view of the surroundings. Reference [52] proposed a fisheye surroundview system and the convolutional neural network $(\mathrm{CNN})$ architecture for moving object segmentation in an autonomous driving environment, running at 15 frames per second at an accuracy of $40 \%$ Intersection over Union (IoU, in approximate terms, an evaluation metric that calculates the area of overlap between the target mask (ground truth) and predicted mask), and $69.5 \%$ mean IoU. 
The deviation in lens geometry from the ideal/nominal geometry will result in image distortion, such that in extreme cases, e.g., ultra-wide lenses employed in fisheye cameras, straight lines in the physical scene may become curvilinear. In photography, the deviations in camera lens geometry are generally referred to as optical distortion, and are commonly categorized as pincushion distortion, barrel distortion, and moustache distortion. Such distortions may introduce an error in the estimated location of the detected obstacles or features in the image. Hence, it is often a require to "intrinsically calibrate" the camera to estimate the camera parameters and rectify the geometric distortions [55]. We present a detailed discussion of the camera intrinsic calibration and the commonly employed method in Section 3.1.1. Further, it is known that the quality (resolution) of images captured by the cameras may significantly affected by lighting and adverse weather conditions, e.g., snow, intense sun glare, rainstorm, hazy weather, et cetera. Other disadvantages of cameras may include the requirement for large computation power while analyzing the image data [20].

Given the above, cameras are a ubiquitous technology that provides high-resolution videos and images, including color and texture information of the perceived surroundings. Common uses of the camera data on AVs include traffic signs recognition, traffic lights recognition, and road lane marking detection. As the camera's performance and the creation of high-fidelity images are highly dependent on the environmental conditions and illumination, image data are often fused with other sensor data such as radar and LiDAR data, to generate reliable and accurate environment perception in AD.

\section{2. $\operatorname{LiDAR}$}

Light Detection and Ranging, or LiDAR, was first established in the 1960s and was widely used in the mapping of aeronautical and aerospace terrain. In the mid-1990s, laser scanners manufacturers produced and delivered the first commercial LiDARs with 2000 to 25,000 pulses per second (PPS) for topographic mapping applications [56]. The development of LiDAR technologies has evolved continuously at a significant pace over the past few decades and is currently one of the cores perception technologies for Advanced Driver Assistance System (ADAS) and AD vehicles. LiDAR is a remote sensing technology that operates on principle of emitting pulses of infrared beams or laser light which reflect off target objects. These reflections are detected by the instrument and the interval taken between emission and receiving of the light pulse enables the estimation of distance. As the LiDAR scans its surroundings, it generates a 3D representation of the scene in the form of a point cloud [20].

The rapid growth of research and commercial enterprises relating to autonomous robots, drones, humanoid robots, and AVs has established a high demand for LiDAR sensors due to its performance attributes such as measurement range and accuracy, robustness to surrounding changes and high scanning speed (or refresh rate)-for example, typical instruments in use today may register up to 200,000 points per second or more, covering $360^{\circ}$ rotation and a vertical field of view of $30^{\circ}$. As a result, many LiDAR sensor companies have emerged and have been introducing new technologies to address these demands in recent years. Hence, the revenue of the automotive LiDAR market is forecasted to reach a total of 6910 million USD by 2025 [57]. The wavelengths of the current state-of-the-art LiDAR sensors exploited in AVs are commonly $905 \mathrm{~nm}$ (nanometers)—safest types of lasers (Class 1), which suffers lower absorption water than for example $1550 \mathrm{~nm}$ wavelength sensors which were previously employed [58]. A study in reference [59] found that the $905 \mathrm{~nm}$ systems can provide higher resolution of point clouds in adverse weather conditions like fog and rains. The $905 \mathrm{~nm}$ LiDAR systems, however, are still partly sensitive to fog and precipitation: a recent study in [60] conveyed that harsh weather conditions like fogs and snows could degrade the performance of the sensor by $25 \%$.

The three primary variants of LiDAR sensors that can be applied in a wide range of applications include 1D, 2D and 3D LiDAR. LiDAR sensors output data as a series of points, also known as point cloud data (PCD) in either 1D, 2D and 3D spaces and the intensity information of the objects. For 3D LiDAR sensors, the PCD contains the $x, y, z$ 
coordinates and the intensity information of the obstacles within the scene or surroundings. For AD applications, LiDAR sensors with 64- or 128- channels are commonly employed to generate laser images (or point cloud data) in high resolution [61,62].

- $1 \mathrm{D}$ or one-dimensional sensors measure only the distance information (x-coordinates) of objects in the surroundings.

- $2 \mathrm{D}$ or two-dimensional sensors provides additional information about the angle (y-coordinates) of the targeted objects.

- $\quad 3 \mathrm{D}$ or three-dimensional sensors fire laser beams across the vertical axes to measure the elevation (z-coordinates) of objects around the surroundings.

LiDAR sensors can further be categorized as mechanical LiDAR or solid-state LiDAR (SSL). The mechanical LiDAR is the most popular long-range environment scanning solution in the field of AV research and development. It uses the high-grade optics and rotary lenses driven by an electric motor to direct the laser beams and capture the desired field of view $(\mathrm{FoV})$ around the $\mathrm{AV}$. The rotating lenses can achieve a $360^{\circ}$ horizontal FoV covering the vehicle surroundings. Contrarily, the SSLs eliminate the use of rotating lenses and thus avoiding mechanical failure. SSLs use a multiplicity of micro-structured waveguides to direct the laser beams to perceive the surroundings. These LiDARs have gained interest in recent years as an alternative to the spinning LiDARs due to their robustness, reliability, and generally lower costs than the mechanical counterparts. However, they have a smaller and limited horizontal FoV, typically $120^{\circ}$ or less, than the traditional mechanical LiDARs $[30,63]$.

Reference [64] compares and analyzes 12 spinning LiDAR sensors that are currently available in the market from various LiDAR manufacturers. In [64], different models and laser configurations are evaluated in three different scenarios and environments, including dynamic traffic, adverse weather generated in a weather simulation chamber, and static targets. The results demonstrated that the Ouster OS1-16 LiDAR model had the lowest average number of points on reflective targets and the performance of spinning LiDARs are strongly affected by intense illumination and adverse weather, notable where precipitation is high and there is non-uniform or heavy fog. Table 3 shows the general specifications of each tested LiDAR sensor in the study of [64] (comprehensive device specifications are presented as well in [65]). In addition, we extended the summarized general specifications in the study of $[64,65]$ with other LiDARs, including Hokuyo $210^{\circ}$ spinning LiDAR and SSLs from Cepton, SICK, and IBEO, and the commonly used ROS drivers for data acquisition from our initial findings.

Laser returns are discrete observations that are recorded when a laser pulse is intercepted and reflected by the targets. LiDARs can collect multiple returns from the same laser pulse and modern sensors can record up to five returns from each laser pulse. For instance, the Velodyne VLP-32C LiDAR analyze multiple returns and reports either the strongest, last, or dual return, depending on the laser return mode configurations. In single laser return mode (strongest return or last return), the sensor analyzes lights received from the laser beam in one direction to determine the distance and intensity information and subsequently employs this information to determine the last return or strongest return. In contrast, sensors in dual return configuration mode will return both the strongest and last return measurements. However, the second-strongest measurements will return as the strongest if the strongest return measurements are like the last return measurements. Not to mention that points with insufficient intensity will be disregarded [66].

In general, at present, 3D spinning LiDARs are more commonly applied in self-driving vehicles to provide a reliable and precise perception of in day and night due to its broader field of view, farther detection range and depth perception. The acquired data in point cloud format provides a dense 3D spatial representation (or "laser image") of the $\mathrm{AVs}^{\text {" }}$ surroundings. LiDAR sensors do not provide color information of the surroundings compared to the camera systems and this is one reason that the PCD is often fused with data from different sensors using sensor fusion algorithms. 


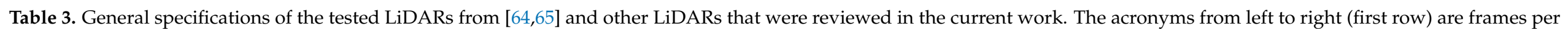

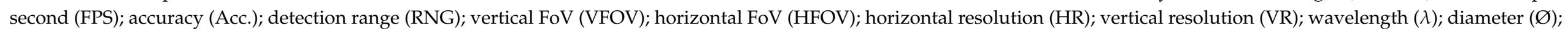

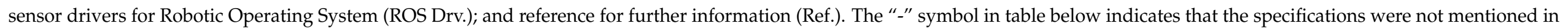
product datasheet.

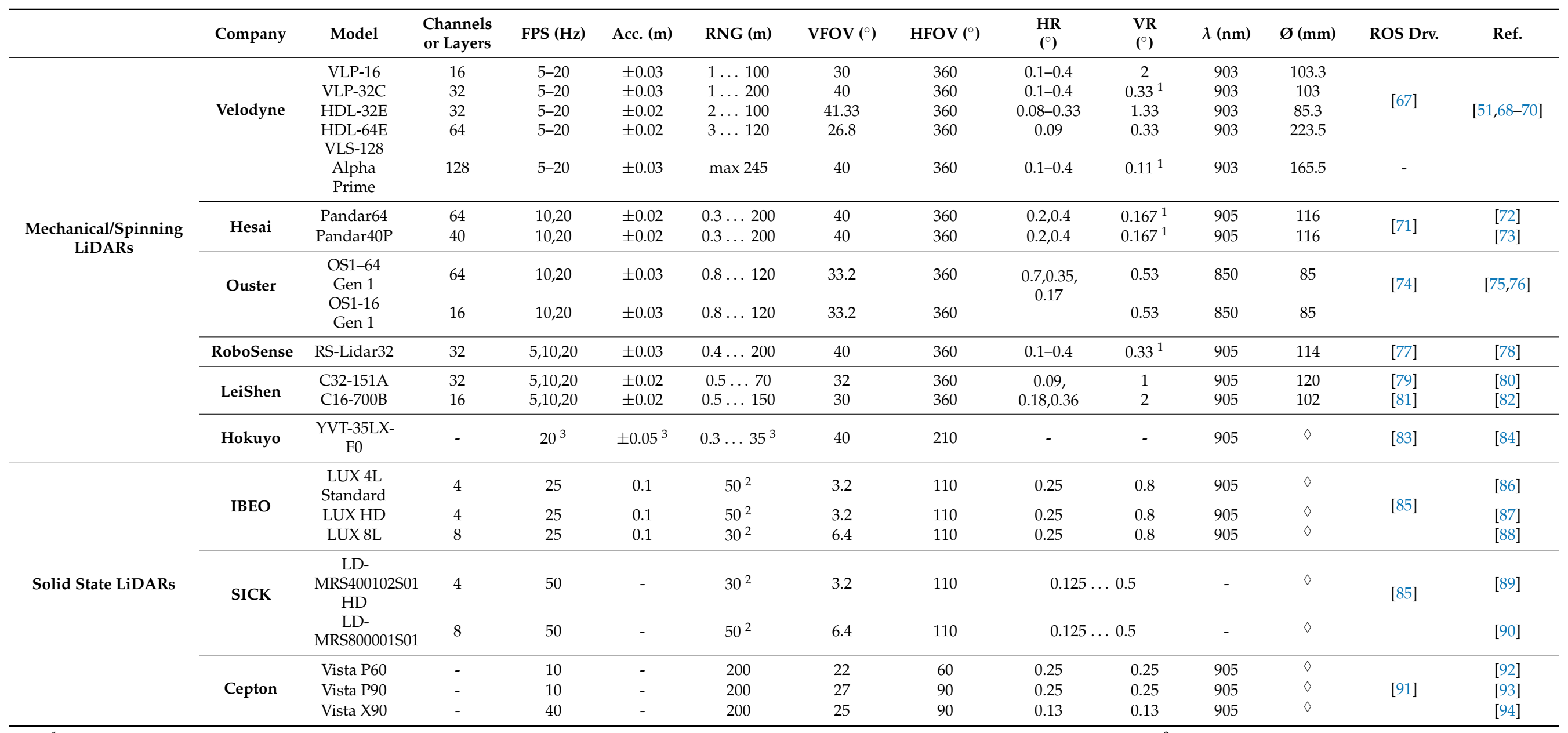

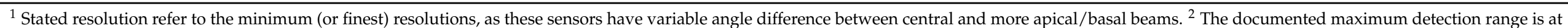

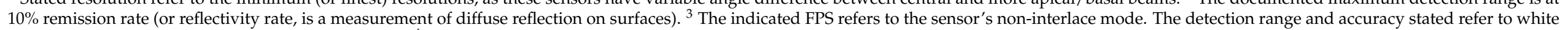
paper detections below $15 \mathrm{~m}$ at center of vertical scan. ${ }^{\diamond}$ Dimension/Size of the sensors are in rectangular shape: width $(\mathrm{W}) \times$ height $(\mathrm{H}) \times$ depth $(\mathrm{D})-$ see individual references for actual dimensions. 


\subsection{Radar}

Radio Detection and Ranging, or Radar, was first established before World War II and operated on the principle of radiating electromagnetic (EM) waves within the area of interest and receiving the scattered waves (or reflections) of targets for further signal processing and establishing range information about the targets. It uses the Doppler property of EM waves to determine the relative speed and relative position of the detected obstacles [30], The Doppler effect, also known as Doppler shift, refers to the variations or shifts in wave frequency arising from relative motion between a wave source and its targets. For instance, the frequency of the received signal increases (shorter waves) when the target moves towards the direction of the radar system [95]. The general mathematical equation of Doppler frequency shift of a radar can be represented as [96,97]:

$$
f_{D}=\frac{2 \times V_{r} \times f}{C}=\frac{2 \times V_{r}}{\lambda}
$$

where $f_{D}$ is the Doppler frequency in Hertz (Hz); $V_{r}$ is the relative speed of the target; $f$ is the frequency of the transmitted signal; $C$ is the speed of light $\left(3 \times 10^{8} \mathrm{~m} / \mathrm{s}\right)$ and $\lambda$ is the wavelength of the emitted energy. In practice, the Doppler frequency change in a radar occurs twice; firstly, when the EM waves are emitted to the target and secondly, during the reflection of the Doppler shifted energy to the radar (source).

Commercial radars available on the market currently operate at $24 \mathrm{GHz}$ (Gigahertz), $60 \mathrm{GHz}, 77 \mathrm{GHz}$, and $79 \mathrm{GHz}$ frequencies. Compared to the $79 \mathrm{GHz}$ radar sensors, $24 \mathrm{GHz}$ radar sensors have a more limited resolution of range, velocity, and angle, leading to problems in identifying and reacting to multiple hazards and are predicted to be phased out in the future [30]. The propagation of the EM waves (radar) is impervious to adverse weather conditions and radar function is independent of the environment illumination conditions; hence, they can operate at day or night in foggy, snowy, or cloudy conditions. Among the drawbacks of radar sensors are the false detection of metal objects around the perceived surroundings like road signs or guardrails and the challenges of distinguishing static, stationary objects [21]. For instance, the difference between an animal carcass (static objects) and the road may pose a challenge for radars to resolve due to the similarity in Doppler shift [98]. Initial findings within the present research using $79 \mathrm{GHz}$ automotive radar sensor (SmartMicro [22]) demonstrated in [22] showed a high frequency of falsepositive detections within the area of interest. Figure 4 shows an example of the falsepositive detections of objects at about 5-7 $\mathrm{m}$ from the mounted sensors.

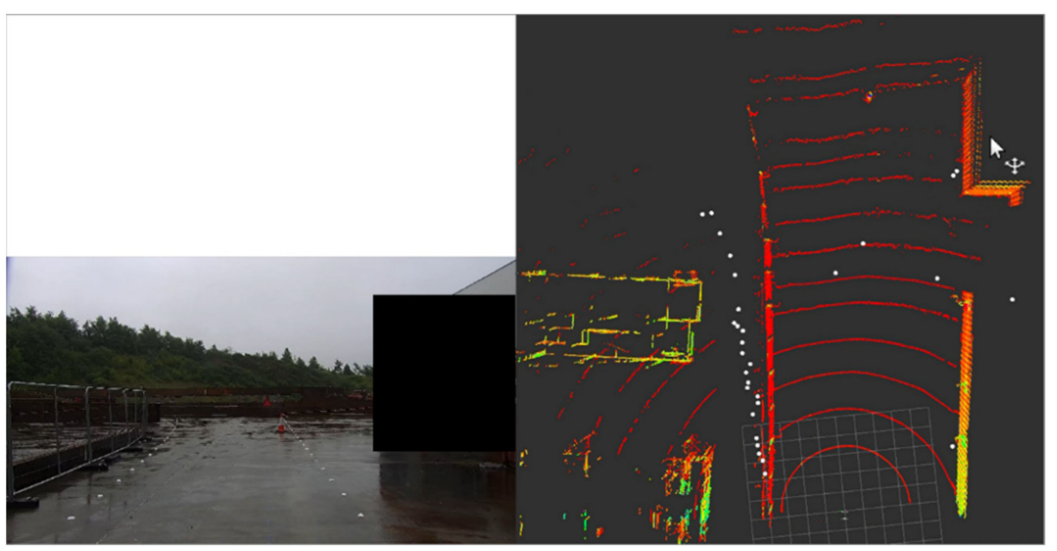

Figure 4. Visualization (before correction for several degrees of sensor misalignment) of false-positive detections in current exploratory research. The colored points in the point clouds visualization represent LiDAR point cloud data and white points represent radar point cloud data. Several falsepositive radar detections are highlighted by the grey rectangle, located at approximately $5-7 \mathrm{~m}$ from the radar sensor. The radar sensor in present setup is in short-range mode (maximum detection range is $19 \mathrm{~m}$ ); hence, the traffic cone located at $20 \mathrm{~m}$ is not detectable. 
Radar sensors in $\mathrm{AD}$ vehicles are commonly integrated invisibly in several locations, such as on the roof near the top of the windshield, behind the vehicle bumpers or brand emblems. It is essential to ensure the precision of mounting positions and orientations of radars in production, as any angular misalignment could have fatal consequences for operation of the vehicle, such errors including false or late detections of obstacles around the surroundings $[99,100]$. Medium-Range Radar (MRR), Long-Range Radar (LRR), and Short-Range Radar (SRR) are the three major categories of automotive radar systems. AV manufacturers utilize SRR for packing assistance and collision proximity warning, MRR for side/rear collision avoidance system and blind-spot detection and LRR for adaptive cruise control and early detection applications [30]. We reviewed the general specifications of several radar sensors from various manufacturers, such as SmartMicro, Continental, and Aptiv Delphi and an overview is presented in Table 4.

Table 4. Summary of the general specifications of radar sensors from SmartMicro, Continental and Aptiv Delphi. The acronyms (first column from top to bottom) are frequency (Freq), horizontal FoV (HFOV), vertical FoV (VFOV), range accuracy (Range Acc), velocity range (Vel Range), input/output interfaces (IO Interfaces) and ROS (Robotic Operating System) drivers for that specific sensors. The "-" symbol in table indicates that the specifications were not mentioned in product datasheet.

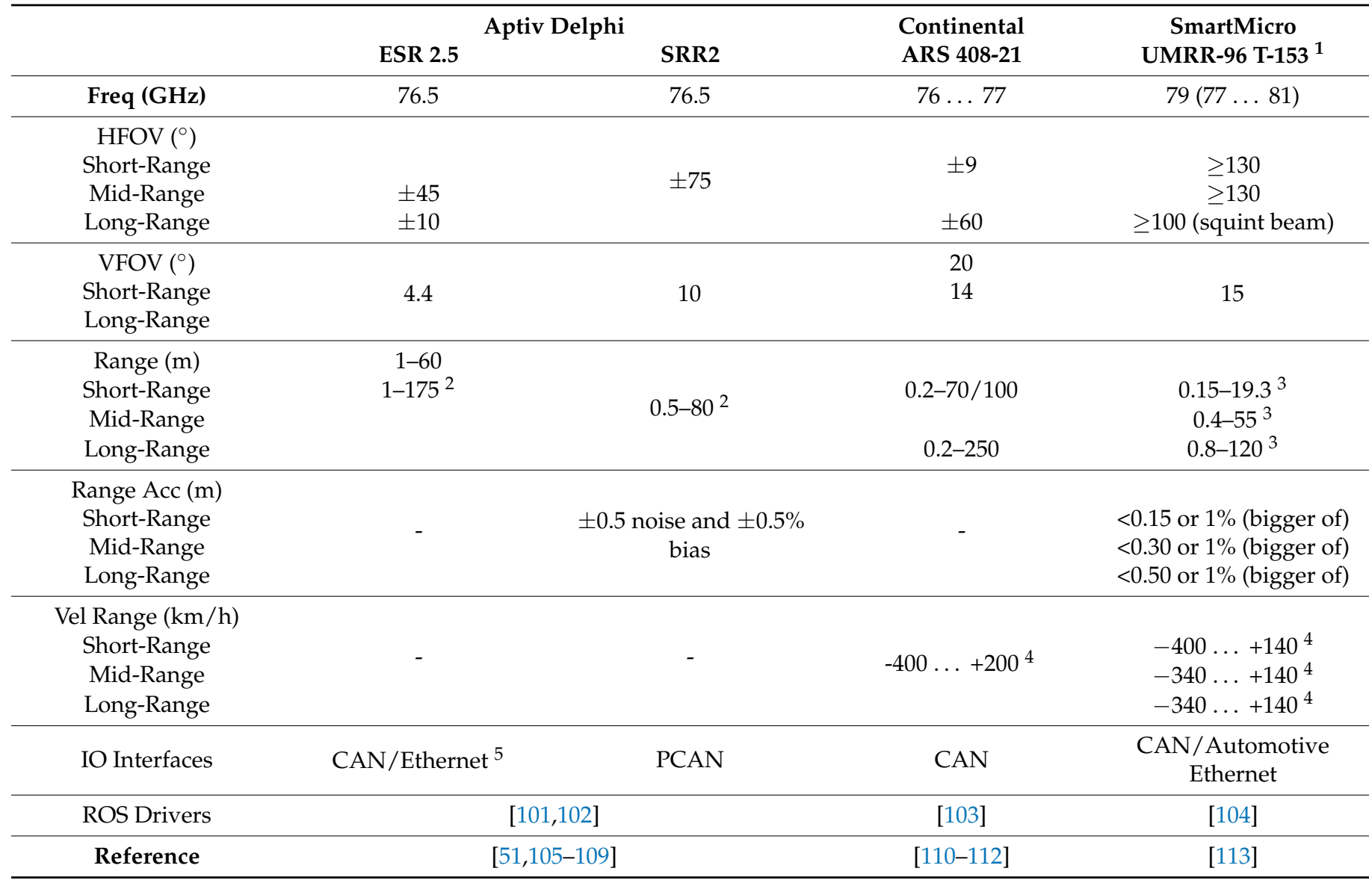

${ }^{1}$ It is recommended to use PCAN-USB adapter from PEAK System for connections of Controller Area Network (CAN) to a computer via Universal Serial Bus (USB) [114]. ${ }^{2}$ Range indicated for ESR 2.5 (long-range mode) and SRR2 is measured at $10 \mathrm{~dB}$ and $5 \mathrm{~dB}$, respectively. ${ }^{3}$ Range may vary depending on the number of targets in the observed environment and will not achieve a $100 \%$ true-positive detection rate. ${ }^{4}$ A negative velocity range indicates the object is moving away from the radar (opening range) and a positive value indicates the object is moving toward the radar (closing range) [115]. ${ }^{5}$ Internet Protocol (IP) address specified on request with a sale unit and is not modifiable by user [116].

In general, radar sensors are one of the well-known sensors in the autonomous systems and are commonly employed in $\mathrm{AVs}$ to provide a reliable and precise perception of obstacles in day and night because of its capability to function irrespective of illumination and adverse weather conditions. It provides additional information, such as speed of the 
detected moving obstacles and can perform mapping in either short, medium, or longrange depending on the configuration mode. The radar sensor, however, is not generally suitable for object recognition applications because of their coarse resolutions compared to cameras. Therefore, AV researchers often fuse radar information with other sensory data, such as camera and LiDAR, to compensate for the limitations of radar sensors.

\section{Sensor Calibration and Sensor Fusion for Object Detection}

According to an article from Lyft Level 5, a self-driving division of Lyft in the United States [117], sensor calibration is one of the least discussed topics in the development of autonomous systems. It is the foundation block of an autonomous system and their constituent sensors, and it is a requisite processing step before implementing sensor fusion techniques and algorithms for AD applications. Sensor calibration notifies the autonomous system about the sensors' position and orientation in real-world coordinates by comparing the relative positions of known features as detected by the sensors. Precise calibrations are vital for further processing steps, such as sensor fusion and implementation of algorithms for obstacle detection, localization and mapping, and control. Further, sensor fusion is one of the essential tasks in AD applications that fuses information obtained from multiple sensors to reduce the uncertainties compared to when sensors are used individually. The fusion algorithms are used principally in the perception block of the overall AD architecture, which involves the object detection sub-processes. Reference [118] presented the MultiSensor Data Fusion (MSDF) framework for AV perception tasks, as depicted in Figure 5. The MSDF framework consists of a sensor alignment process and several object detection processing chains, and subsequently integrates the outputs from sensor alignment and object detection for further processing tasks.

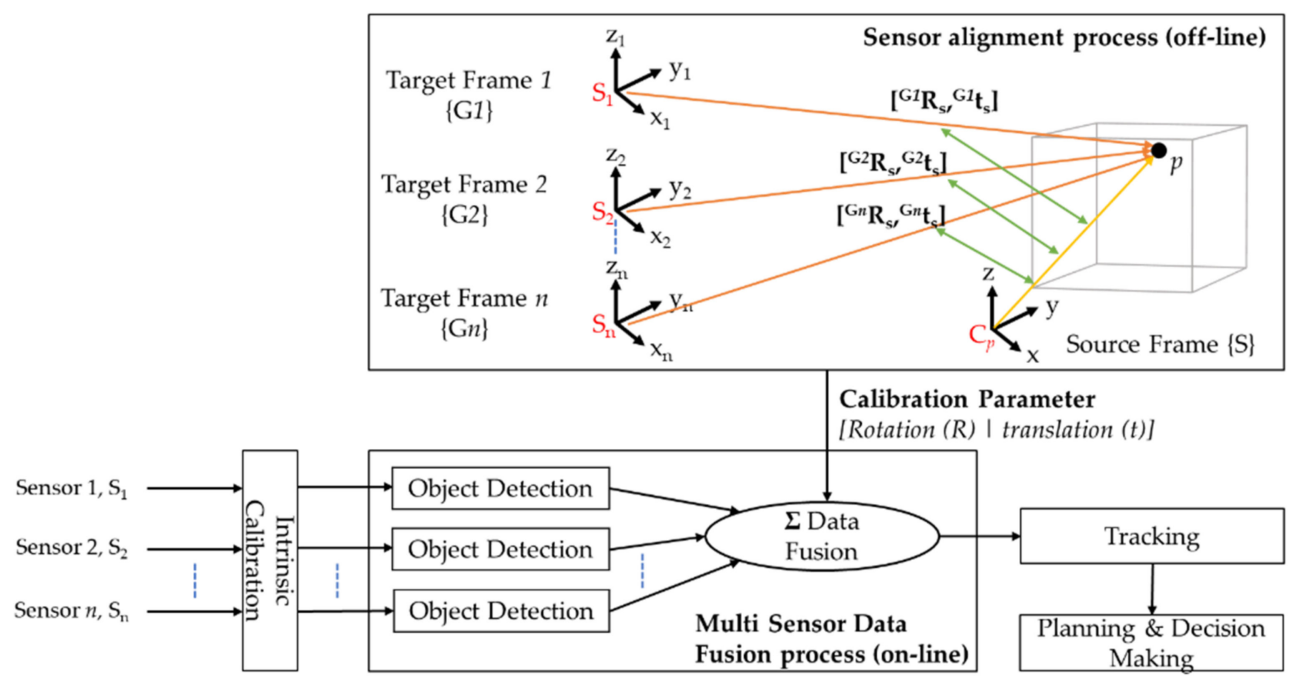

Figure 5. The structure of Multi-Sensor Data Fusion (MSDF) framework for $n$ given sensors. It consists of a sensor alignment process (estimation of calibration parameters-rotation matrix and translations vector) and an object detection process which contains $n$ processing chains, each provides a list of the detected obstacles. Figure redrawn based on depictions in [118], but with the inclusion of an intrinsic calibration process.

Section 3.1 highlights the three categories of calibrations: intrinsic calibration, extrinsic calibration, and temporal calibration [119] and provides an overview of an existing calibration packages which has been employed in the current research. Section 3.2 reviews the three sensor approaches, namely high-level fusion (HLF), low-level fusion (LLF), and mid-level fusion (MLF) for object detection and summarizes the commonly employed algorithms, followed by the challenges of sensor fusion for safe and reliable environment perception. 


\subsection{Sensor Calibrations}

\subsubsection{Intrinsic Calibration Overview}

Intrinsic calibration addresses sensor-specific parameters and is conducted before implementing extrinsic calibration and obstacle detection algorithms. Intrinsic calibration estimates the internal or intrinsic parameters of a sensor, e.g., focal lengths of a vision camera, which correct for systematic or deterministic aberrations (errors). These parameters are anticipated to be consistent once the intrinsic parameters are estimated [120]. It is known through personal communication that Velodyne LiDARs are calibrated to $10 \%$ reflectivity of the National Institute of Standards and Technology (NIST) targets. Therefore, the reflectance of the obstacles below the $10 \%$ reflectivity rate may not be detected by the LiDAR [121]. Algorithms and methods for intrinsic calibration of sensors have received considerable attention with significant advancement over the last number of years and now, are well-established in the literature. These algorithms and methodologies may vary from one sensor to another [122-129]. This subsection aims to provide an overview of the most used calibration targets and the calibration methodologies for the pinhole camera model.

The pinhole camera model is a well-known and commonly used model (inspired by the simplest cameras [130]) in computer vision applications, which describes the mathematical relationship of the projection of points in 3D space on to a 2D image plane [131]. Figure 6 visualizes the camera pinhole model, which consists of a closed box with a small opening (pinhole) on the front side through which the light rays from a target enters and produces an image on the opposing camera wall (image plane) [132].

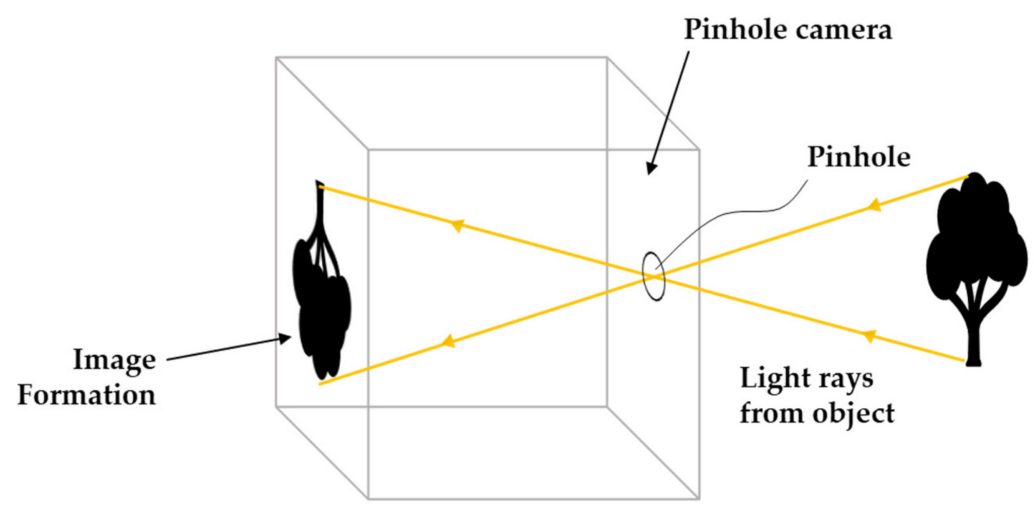

Figure 6. A graphical representation of the pinhole camera. The pinhole (aperture) restraints the light rays from the target from entering the pinhole; hence, affecting the brightness of the captured image (during image formation). A large pinhole (a wide opening) will result in a brighter image but is less clear due to blurriness on both background and foreground. Figure redrawn based on depictions in $[132,133]$.

From a mathematical perspective (Figure 7), the model involves a 3D camera coordinate system and a 2D image coordinate system to calibrate the camera using a perspective transformation method $[134,135]$. The calibration process involves utilizing the extrinsic parameters $(a 3 \times 4$ matrix that consists of the rotation and translation [R $\mid t]$ transformation) to transform the $3 \mathrm{D}$ points in world coordinate space $\left(X_{W}, Y_{W}, Z_{W}\right)$ into their corresponding $3 \mathrm{D}$ camera coordinates $\left(X_{C}, Y_{C}, Z_{C}\right)$. In addition, it involves employing the intrinsic parameters (also referred to as the $3 \times 3$ intrinsic matrix, $K$ [136]), to transform the $3 \mathrm{D}$ camera coordinates into the 2D image coordinates $(x, y)$. 


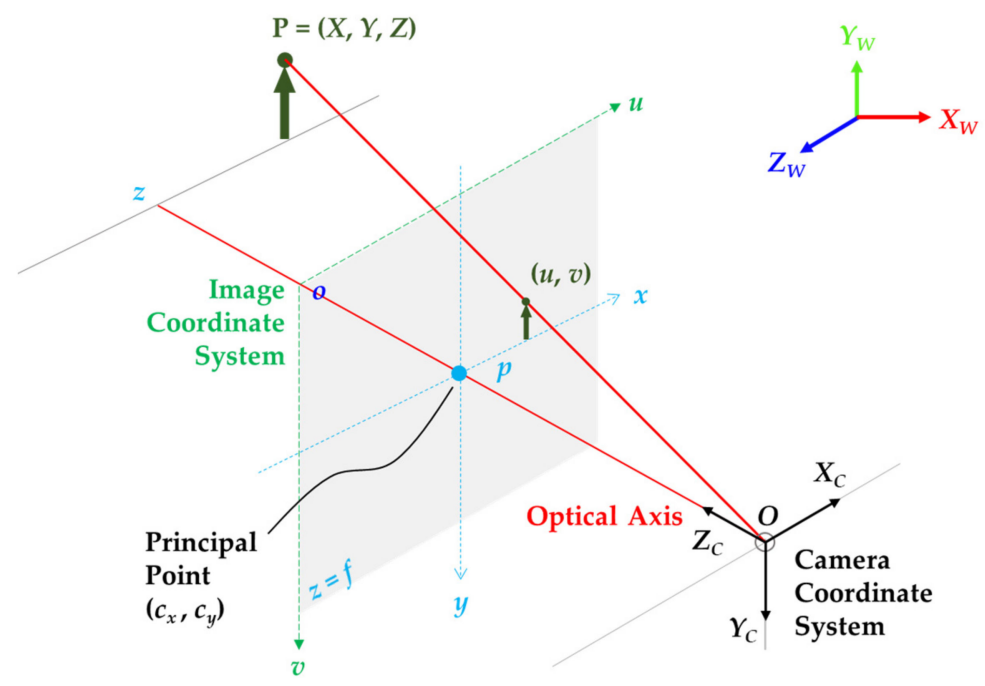

Figure 7. The pinhole camera model from a mathematical perspective. The optical axis (also referred to as principal axis) aligns with the $Z$-axis of the camera coordinate system $\left(Z_{C}\right)$, and the intersections between the image plane and the optical axis is referred to as the principal points $\left(c_{x}, c_{y}\right)$. The pinhole opening serves as the origin $(O)$ of the camera coordinate system $\left(X_{C}, Y_{C}, Z_{C}\right)$ and the distance between the pinhole and the image plane is referred to as the focal length $(f)$. Computer vision convention uses right-handed system with the $z$-axis pointing toward the target from the direction of the pinhole opening, while $y$-axis pointing downward, and $x$-axis rightward. Conventionally, from a viewer's perspective, the origin (o) of the $2 \mathrm{D}$ image coordinate system $(x, y)$ is at the top-left corner of the image plane with $x$-axis pointing rightward, and $y$-axis downward. The $(u, v)$ coordinates on the image plane refers to the projection of points in pixels. Figure redrawn based on depictions in $[125,134,135]$.

The perspective transformation method outputs a $4 \times 3$ camera matrix $(P)$, also referred to as the projection matrix, which consists of the intrinsic and extrinsic parameters to transform 3D world coordinate space into the 2D image coordinates. It should be stressed that the extrinsic calibration parameters in the camera calibration context differ from the extrinsic calibration process of one or more sensors relative to another sensor. It is known that the camera matrix does not account for any lens distortion-the ideal pinhole camera lacking a lens. The general mathematical equation of the perspective method is represented as $[125,134,137,138]$ :

$$
P=K[\mathrm{R} \mid \mathbf{t}] \text { or } P=\left[\begin{array}{ccc}
f_{x} & s & c_{x} \\
0 & f_{y} & c_{y} \\
0 & 0 & 1
\end{array}\right]\left[\begin{array}{llll}
r_{11} & r_{12} & r_{13} & t_{1} \\
r_{21} & r_{22} & r_{23} & t_{2} \\
r_{31} & r_{32} & r_{33} & t_{3}
\end{array}\right]\left[\begin{array}{c}
X_{w} \\
Y_{w} \\
Z_{w} \\
1
\end{array}\right]
$$

where $P$ is the $4 \times 3$ camera matrix; $[R \mid t]$ represents the extrinsic parameters (rotation and translation) to transform the 3D world points $\left(X_{W}, Y_{W}, Z_{W}\right)$ into camera coordinates; and $K$ is the intrinsic matrix of the pinhole camera that consists of the geometry properties of a camera, such as axis skew (s), optical centers or principal points offset $\left(c_{x}, c_{y}\right)$ and focal lengths $\left(f_{x}, f_{y}\right)$. The focal length $(f)$ of a camera refers to the distance between the pinhole and the image plane and it determines the projection scale of an image. Hence, a smaller focal length will result in a smaller image and a larger viewing angle [132]. A detailed discussion of the projection of 3D world points into a 2D image plane, estimation of camera lens distortion, and the implementations are beyond the scope of this paper (see $[132,133]$ for a more comprehensive overview).

Camera calibration (or camera re-sectioning [137]) is the process of determining the intrinsic and extrinsic parameters that comprise the camera matrix. Camera calibration is one of the quintessential issues in computer vision and photogrammetry and has re- 
ceived considerable attention over the last number of years. A variety of calibration techniques, [124-126,133,139-142] to cite a few, have been developed to accommodate various applications, such as AVs, Unmanned Surface Vehicle (USV) or underwater 3D reconstructions. Reference [141] classified these techniques into:

- Photogrammetric calibration. This approach uses the known calibration points observed from a calibration object (usually a planar pattern) where the geometry in the $3 \mathrm{D}$ world space is known with high precision.

- Self-calibration. This approach utilizes the correspondence between the captured images from a moving camera in a static scene to estimate the camera intrinsic and extrinsic parameters.

The well-known Zhang method is one of the most used camera calibration techniques. It uses a combination of photogrammetric calibration and self-calibration techniques to estimate the camera matrix. It uses the known calibration points observed from a planar pattern (Figure 8) from multiple orientations (at least two) and the correspondence between the calibration points in various positions to estimate the camera matrix. In addition, the Zhang method for camera calibration does not require the motion information when either the camera or the planar pattern are moved relative to each other [141].

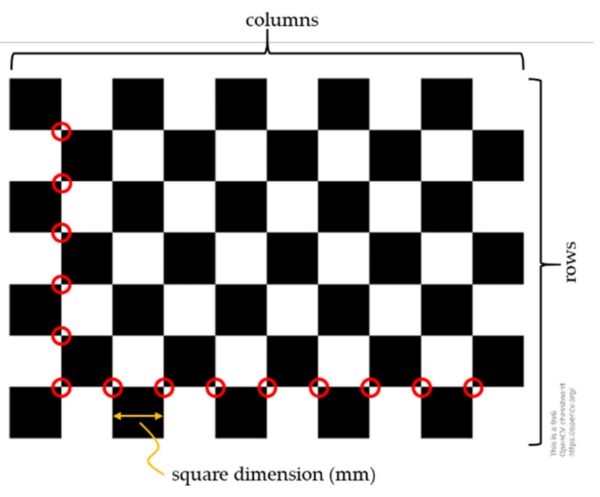

(a)

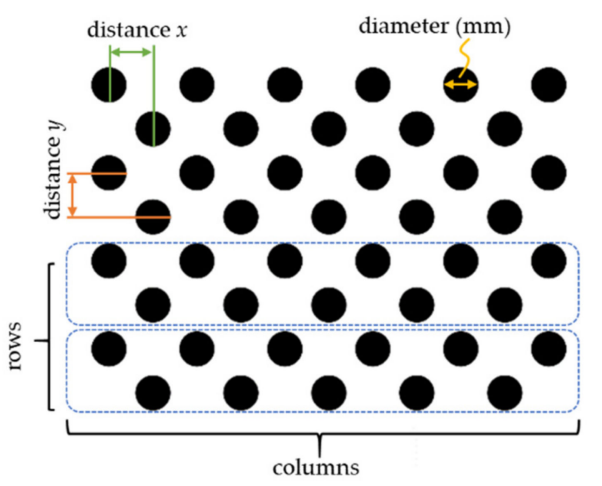

(b)

Figure 8. The most employed patterns for camera calibration. (a) A 7 rows $\times 10$ columns checkerboard pattern. The calibration uses the interior vertex points of the checkerboard pattern; thus, the checkerboard in (a) will utilize the $6 \times 9$ interior vertex points (some of which are circled in red) during calibration. (b) A 4 rows $\times 11$ columns asymmetrical circular grid pattern. The calibration uses the information from circles (or "blobs" in image processing terms) detection to calibrate the camera. Other planar patterns include symmetrical circular grid and ChArUco patterns (a combination of checkerboard pattern and ArUco pattern) $[128,137,141]$. Figures source from OpenCV and modified.

The popular open source "camera_calibration" package in ROS offers several preimplemented scripts to calibrate monocular, stereo, and fisheye cameras using the planar pattern as a calibration target. The calibration result includes the intrinsic matrix of a distorted image, distortion parameters, rectification matrix (stereo cameras only), camera matrix or projection matrix, and other operational parameters such as binning and region of interest (ROI). The calibration package was built based on the OpenCV camera calibration and 3D reconstruction package. Further, the calibration algorithm was implemented based on the well-known Zhang method and the camera calibration toolbox for MATLAB by Bouguet, J.Y. [128,134].

In general, camera calibration results are no longer applicable if the camera's zoom (focal length) has changed. It should be noted that in our experience, radar and LiDAR sensors are factory intrinsic-calibrated.

\subsubsection{Extrinsic Calibration Overview}

Extrinsic calibration is a rigid transformation (or Euclidean transformation) that maps the points from one 3D coordinate system to another, for example, a rigid transformation 
of points from the 3D world or 3D LiDAR coordinate system to the 3D camera coordinate system. The extrinsic calibration estimates the position and orientation of the sensor relative to the three orthogonal axes of 3D space (also known as the 6 degrees of freedoms, $6 \mathrm{DoF}$ ) with respect to an external frame of reference $[119,143]$. The calibration process outputs the extrinsic parameters that consist of the rotation $(R)$ and translation $(t)$ information of the sensor and is commonly represented in a $3 \times 4$ matrix, as shown in Equation (2). This section aims to provide a comparative overview of existing open-source multi-sensor extrinsic calibration packages and a summary of algorithms proposed in the literature for extrinsic calibration of camera, LiDAR, and radar sensors comprising a sensor fusion system.

The studies of extrinsic calibration and the methodologies are well-established in the literature, see reference [143-151] for example. Though, the extrinsic calibration of multiple sensors with various physical measurement principles can pose a challenge in multi-sensor systems. For instance, it is often challenging to match the corresponding features between camera images (dense data in pixels) and 3D LiDAR or radar point clouds (sparse depth data without color information) [144]. The target-based extrinsic calibration approach employs specially designed calibration targets, such as marker-less planar pattern [51], checkerboard pattern [145], orthogonal and trihedral reflector [51,143, $146,148]$, circular pattern to calibrate multiple sensor modalities in autonomous systems. The targetless extrinsic calibration approach leverages the estimated motion by individual sensors or utilizes the features in the perceiving environment to calibrate the sensors. However, employing the perceived environment features requires multimodal sensors to extract the same features within the environment and is sensitive to the calibration environment $[144,149]$.

A comparative overview of existing extrinsic calibration tools in [146] reported that the available tools only addressed pairwise calibrations of a maximum of two sensing modalities. For instance, the framework presented in [143] uses a coarse to fine extrinsic calibration approach to calibrate the RGB camera with a Velodyne LiDAR. The algorithm utilizes a novel 3D marker with four circular holes to estimate the coarse calibration parameters and further refine these parameters using the dense search approach to estimate a more accurate calibration in the small $6 \mathrm{DoF}$ calibration parameters subspace. Reference [150] presented an extrinsic calibration algorithm which utilizes the Planar Surface Point to Plane and Planar Edge to back-projected Plane geometric constraints to estimate the extrinsic parameters of the 3D LiDAR and a stereo camera using a marker-less planar calibration target. As highlighted in the previous paragraph, each sensing modality has a different physical measurement principle; thus, sensor setups with more modalities may duplicate the calibration efforts, especially in mobile robots in which sensors are frequently dismounted or repositioned. For this reason, reference $[145,148]$ presented a novel calibration method to extrinsically calibrate all three sensing modalities, namely radar, LiDAR, and camera with a specially designed calibration target. Table 5 below summarizes the open-source extrinsic sensor calibration tools, specifically for camera, LiDAR sensor, and radar sensor extrinsic calibration. 
Table 5. An overview of the available open-source extrinsic sensor calibration tools for multi-sensing modalities, specifically for LiDAR, radar, stereo camera, and monocular camera. The acronyms of the columns (from left to right) are the referenced literature (Ref), stereo camera (S), monocular camera (M), LiDAR (L) and Radar (R). The platform and toolbox column refer to the working environment of the toolbox and a reference link to the open-source calibration toolbox. Further, the calibration target column summarizes the calibration target used for extrinsic sensor calibration. The symbols $\checkmark$ and $x$ indicate whether the proposed open-source toolbox can calibrate a particular sensor. The "** symbol indicates that the proposed calibration tool claims to support monocular camera calibration. The " $\sim$ " symbol indicates that a stereo camera could be calibrated as two separate monocular cameras, but in principle, it is suboptimal. The "_ symbol indicates that the extrinsic calibration tool is not mentioned or openly or freely available to the research community. Based on [145] with modification.

\begin{tabular}{|c|c|c|c|c|c|c|c|}
\hline Ref & $\mathrm{S}$ & $\mathbf{M}$ & $\mathbf{L}$ & $\mathbf{R}$ & Platform & Toolbox & Calibration Target \\
\hline$[145]^{1}$ & $\checkmark$ & * & $\checkmark$ & $\checkmark$ & ROS & [146] & $\begin{array}{l}\text { Styrofoam planar with four circular holes } \\
\text { and a copper plate trihedral } \\
\text { corner reflector. }\end{array}$ \\
\hline [148] & $\sim$ & $\checkmark$ & $\checkmark$ & $\checkmark$ & - & - & $\begin{array}{l}\text { Checkerboard triangular pattern with } \\
\text { trihedral corner retroreflector. }\end{array}$ \\
\hline [152] & $\checkmark$ & $x$ & $\checkmark$ & $x$ & MATLAB & [153] & LiDARTag $^{2}$ and AprilTag ${ }^{2}$ \\
\hline$[154]^{3}$ & $\checkmark$ & * & $\checkmark$ & $x$ & ROS & [155] & $\begin{array}{c}\text { Planar with four circular holes and four } \\
\text { ArUco markers }{ }^{4} \text { around the } \\
\text { planar corners. }\end{array}$ \\
\hline [156] & $\checkmark$ & * & $\checkmark$ & $x$ & ROS & [157] & $\begin{array}{l}\text { ArUco marker on one corner of the hollow } \\
\text { rectangular planar cardboard marker. }\end{array}$ \\
\hline [143] & $\sim$ & $\checkmark$ & $\checkmark$ & $x$ & ROS & [158] & 3D marker with four circular holes pattern. \\
\hline [159] & $\sim$ & $\checkmark$ & $\checkmark$ & $x$ & ROS & [160] & Planar checkerboard pattern. \\
\hline
\end{tabular}

1 The toolbox binds with the commonly employed ROS and includes a monocular camera detector for extrinsic calibration, but reported results relate to stereo camera only [145]. ${ }^{2}$ LiDARTag (point clouds) and AprilTag (images) is a visual fiducial tag (QR-code like pattern). ${ }^{3}$ The extrinsic calibration tool is an enhancement version of the previous work from [161]. ${ }^{4}$ ArUco marker is a synthetic $2 \mathrm{D}$ square marker with a wide black border and an inner binary matrix.

Reference [145] proposed a novel extrinsic calibration tool that utilizes a target-based calibration approach and a joint extrinsic calibration method to facilitate the extrinsic calibration of three sensing modalities. The proposed calibration target design consists of four circular, tapered holes centrally located within a large rectangular board and a metallic trihedral corner reflector located between the four circles at the rear of the board (Figure 9). The corner reflector provides a strong radar reflection as the Styrofoam board is largely transparent to electro-magnetic radiation. Additionally, the circular edges provide an accurate and robust detection for both LiDAR (especially when intersecting with fewer LiDAR beams) and camera. The authors of this system established three possible optimization configurations for joint extrinsic calibration, namely:

- $\quad$ Pose and Structure Estimation (PSE). It estimates the latent variables of the true board locations and optimizes the transformations to a precise estimate of all calibration target poses employing the estimated latent variables.

- Minimally Connected Pose Estimation (MCPE). It relies on a reference sensor and estimates the multi-sensing modalities transformations to a single reference frame.

- Fully Connected Pose Estimation (FCPE). It estimates the transformations between all sensing modalities "jointly" and enforces a loop closure constraint to ensure consistency. 


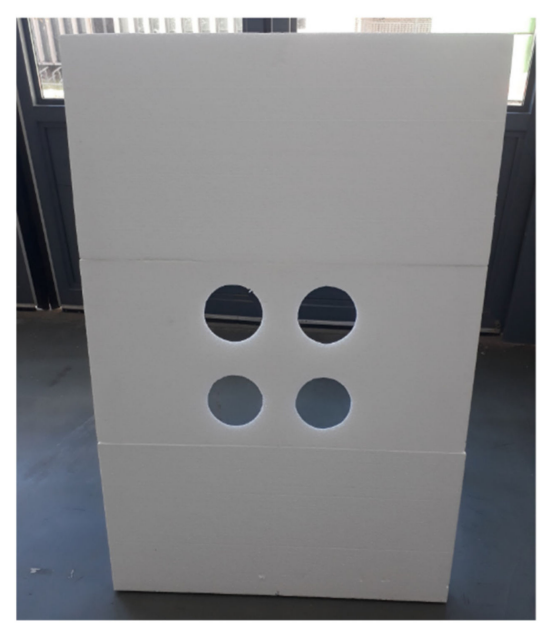

(a)

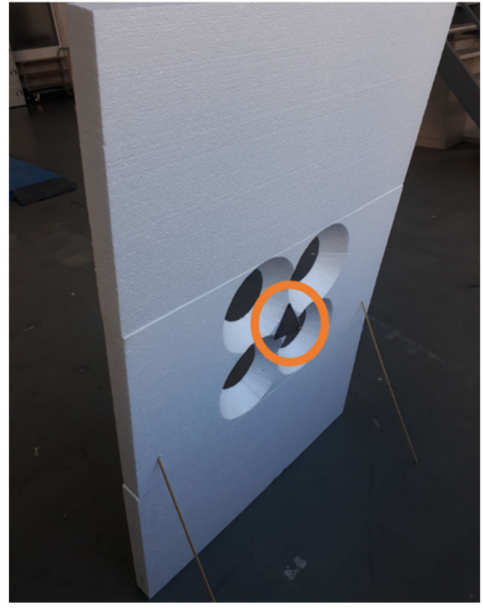

(b)

Figure 9. The proposed calibration target design to jointly extrinsic calibrate multiple sensors (radar, camera, LiDAR). It consists of four circulars, tapered holes centrally located within a large rectangular board at the (a) front of the board, and a metallic trihedral corner reflector (circled in orange) located between the four circles at the (b) rear of the board. Figure source from [146,147] and modified.

The proposed calibration tool [146] has bindings with the commonly employed ROS middleware and provides the joint optimization configurations to estimate the sensor poses from simultaneous calibration board detection in multiple locations. It outputs a transformation matrix $(P)$ that can be used to transform the detections from the source reference frame to target reference frame and the poses of the sensor with respect to the parent link for visualization (in ROS). They compared the PSE, MCPE, and FCPE joint optimization results based on multiple variables, such as the required number of calibration board locations and the MCPE reference sensor selections. The results demonstrate that the FCPE joint optimization provided better performance than both MCPE and PSE when employing more than five board locations. A detailed discussion of each joint optimization configuration and its algorithm, and the geometry of the calibration board are beyond the scope of this paper (see $[146,147]$ for a more comprehensive overview).

The current authors utilized and reviewed the calibration tool from reference [146] to extrinsic calibrate the Velodyne VLP-32C LiDAR sensor, SmartMicro UMRR-96 T-153 radar sensor, and Falcon-IQ EZIP-T030(E) Internet Protocol (IP) industrial zoom monocular camera in an initial multi-sensor setup [22]. Observations and recommendations arising from this work include:

- Ensure that the edges of the circles have sufficient contrast with the background, specifically when calibrating the cameras outdoors as was necessary in our case. Though, it is recommended in [146] that calibration of sensors be done indoors to avoid strong wind which may overturn the calibration board.

- Ensure that the camera lenses are protected from rain droplets to reduce noise when calibrating the sensors outdoors, particularly during rainy and blustery weather conditions.

- Additional or modified scripts may be required to match the ROS sensor message types of the board detector nodes depending on the employed ROS sensor drivers. For instance, a Continental ARS430 radar was utilized in [146] and exploited the AutonomouStuff-provided ROS messages which output the detections in an AutonomouStuff sensor message array format [101]. However, the ROS driver from SmartMicro radars outputs the detections in a ROS sensor message type of PointCloud2 format [113]. Table 6 summarizes the sensor message types for each board detector node (as input requirements) of the extrinsic calibration tool.

- Ensure that the edges of the four circles are detected (covered) with sufficient points within the LiDAR point cloud. We examined and compared the elevation angles of 
the Velodyne VLP-32C with the Velodyne HDL-64E ([162], utilized in [146]). The results indicated that the vertical laser points of HDL-64E are distributed uniformly between $-24.9^{\circ}$ to $2^{\circ}$. In comparison, the vertical laser points of Velodyne VLP-32C are concentrated in the middle of the optical center between $-25^{\circ}$ to $15^{\circ}$, as shown in Figure 10. Hence, the position and orientation of the lidar relative to the calibration board may have a significant effect on reported location of circles detected within the lidar data.

- It is suggested in [146] to position the calibration board in a spacious area and capture at least ten calibration board locations in the FoV of all sensors. However, it is not recommended to hold the calibration board, which can affect the detections of the corner reflector (by the radar sensor).

- The stereo camera employed in [146] was constructed from two monocular cameras; namely IDS Imaging UI-3060CP Rev. 2; and exploited the "stereo_image_proc" module in ROS [39] to create the disparity image of the perceived surroundings.

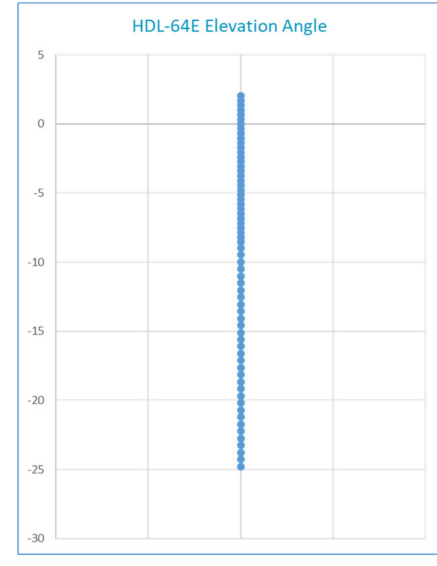

(a)

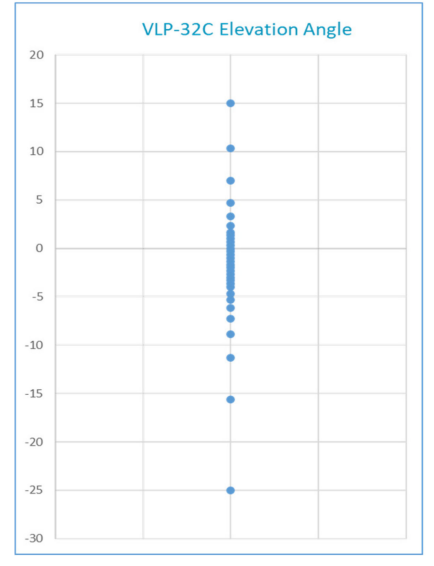

(b)

Figure 10. A graphical representation of the vertical laser points of the (a) Velodyne HDL-64E and the (b) Velodyne VLP-32C. Reference [145] utilizes the Velodyne HDL-64E which consists of 64 channels (layers), and the vertical laser beams are distributed uniformly across the vertical FoV between $-24.9^{\circ}$ to $2^{\circ}$. The initial sensor configurations employed by the current authors [22] employs the Velodyne VLP-32C which consists of 32 channels (or layers) where the vertical laser beams are concentrated in the middle of the optical center across the vertical FoV between $-25^{\circ}$ to $15^{\circ}$. Based on sensor user manual [68].

Table 6. An overview of the ROS topic message types as input requirements for each calibration board detector node, namely monocular camera detector (mono_detector), LiDAR detector (lidar_detector), stereo camera detector (stereo_detector), and radar detector (radar_detector). Based on reference [145,152]. A detailed overview of the ROS sensor message types is available in reference [163].

\begin{tabular}{|c|c|c|}
\hline Detector & Subscribed Topic Name & ROS Sensor Message Types \\
\hline LiDAR & /velodyne_points & sensors_msgs::PointCloud2 \\
\hline Stereo & $\begin{array}{c}\text { /ueye/left/image_rect_color } \\
\text { /ueye/left/camera_info } \\
\text { /ueye/right/camera_info } \\
\text { /ueye/disparity }\end{array}$ & $\begin{array}{c}\text { sensor_msgs::Image } \\
\text { sensor_msgs::CameraInfo } \\
\text { sensor_msgs::CameraInfo } \\
\text { stereo_msgs::DisparityImage }\end{array}$ \\
\hline Monocular & $\begin{array}{l}\text { /ueye/left/image_rect_color } \\
\text { /ueye/left/camera_info }\end{array}$ & $\begin{array}{c}\text { sensor_msgs::Image } \\
\text { sensor_msgs::CameraInfo }\end{array}$ \\
\hline Radar & /radar_converter/detections & radar_msgs::RadarDetectionArray ${ }^{1}$ \\
\hline
\end{tabular}

${ }^{1}$ AutonomouStuff, an automotive platform that offers solutions for developing and deploying AD applications, who provide the generic radar output messages that are not currently available in the commonly employed ROS sensor messages module (deprecated in latest version of ROS1). 
Based on this revision of extrinsic calibration tools available to the research community, it is noticed that most of them addressed only pairwise calibrations of two sensing modalities, with the notable exception of extrinsic calibration tool described in [145] which facilitates joint extrinsic calibration of more than two sensing modalities (radar, camera, and LiDAR) and has bindings with ROS middleware. Other open source extrinsic calibration tools include Kalibr that provides multiple camera calibration or camera-IMU extrinsic calibration and Calirad, that facilitates the extrinsic calibration and temporal calibration of the radar, camera, and LiDAR sensors. It is emphasized again that individual sensors are intrinsic calibrated before implementing extrinsic calibration.

In contrast to target-based extrinsic calibration methods, targetless extrinsic calibration approach methods estimate motion of the sensors or features in the perceiving surroundings, such as road markings to determine extrinsic calibration of the sensors.

\subsubsection{Temporal Calibration Overview}

Temporal calibration is the process of determining the synchronicity (or relative time delay) of multiple sensor data streams with potentially different frequencies and latencies in a multi-sensor setup [119]. For instance, the camera usually captures images at 30 FPS or less, while a LiDAR sensor may scan at a rate as low as $5 \mathrm{~Hz}$. One approach of synchronizing the sensor data is to establish the closest match between the message header timestamps obtained at endpoints (computer). However, in principle, synchronization based on message timestamps is suboptimal because sensors may have unknown latencies, such as communication transfer delays or pre-processing delays in the sensor circuitry [118]. These unknown latencies may not be determinable directly and will likely differ from one sensor to another. The approximate time synchronizer method in the ROS message filter module [164] matches the messages from each sensing modality (or topic in ROS term) based on their header timestamps as a means of time synchronization using an adaptive algorithm. The adaptive algorithm first determines the latest message among the heads of the topic-specific queues as a reference point, and approximately synchronize these messages based on the estimated reference point and within a given threshold.

We utilized the approximate time synchronizer method in [164] to synchronize the sensor data in an initial multi-sensor setup [22]. The results demonstrated that an average of 86.6 per cent of sensor messages with varying frequency of operation were synchronized within a threshold of 50 milliseconds. Further, the most prolonged unsynchronized periods between the camera and LiDAR were found to be 850 milliseconds; between LiDAR and radar, it was 870 milliseconds; and between camera and radar, it was 880 milliseconds. Another synchronization method based on messages header timestamps in ROS is the exact time synchronizer [164], which requires the incoming messages to have an exact timestamp for synchronization. A comprehensive overview of the adaptive algorithm employed in the approximate time synchronizer method and the usage of the methods are beyond the scope of this paper (see [164] for a more detailed overview).

Temporal calibration is often overlooked and is crucial in multi-sensor fusion applications, such as self-driving vehicles which must perform complex sensing and estimation tasks in real-time, such as state estimations and obstacle detections [118]. There are two approaches to temporally calibrate the sensors: external synchronization that utilizes external hardware for time synchronization and internal synchronization, exploiting the attaching timestamps on each sensor measurement for synchronization $[165,166]$. The external synchronization approach uses a central hardware clock as an external source of time or a reference clock to temporal-synchronize the sensors and is precisely relatable to a real-time standard such as Universal Time Coordinated (UTC) standard time. For instance, reference [167] utilizes an external Novatel SMART6-L Global Positioning System (GPS) as a reference clock and exploits the GPS timestamps information to synchronize the system (or computer) clock. Conversely, the internal synchronization approach synchronizes the sensors based on the associated timestamps without the external source of time to obtain a consistent view of time across all sensor networks. Reference [168] proposes the passive 
synchronization algorithms to determine the time offsets when the device and sensor clocks drift, and can significantly reduce the synchronization error, even in the presence of an unknown latency and for sensors with significant clock errors.

A complete sensor-to-sensor calibration, also known as the spatial-temporal calibration, involves extrinsic calibration of the sensors to a unified coordinate space and temporal calibration to estimate the relative time delays between sensor data streams. Reference [169] presents a spatial-temporal calibration method that uses the estimated continuous-time moving object trajectories from Gaussian Processes (GPs) and a target-based approach to calibrate the sensors relative to one another. It utilizes estimated object velocities to estimate relative time delays between sensors. These [169] experiments demonstrated that the proposed algorithm could reliably determine the time delays up to a fraction of the fastest sensor sampling rate. The implementation of the method proposed in [169] has been open sourced in [170] and it has bindings with ROS middleware. Additionally, it applies to any multi-sensor setup once the employed multi-sensor can determine the $3 \mathrm{D}$ position of a moving "target". An insightful discussion of the employed GP algorithms is beyond the scope (see [169-171] for a more comprehensive overview). Further, through personal communication, the target detections become unstable from six meters or more depending on the tracker size. The materials from which the calibration tracker is constructed include (Figure 11) [172]:

- Styrofoam or cardboard to fabricate the triangular planar pattern,

- Printed AprilTag marker with a size of approximately $17 \mathrm{~cm}$ in length, located at the front of the triangular planar and,

- Cardboards to assemble a trihedral corner reflector where the three inner sides of the reflector are overlaid with aluminum foil and attached at the rear of the triangular planar.

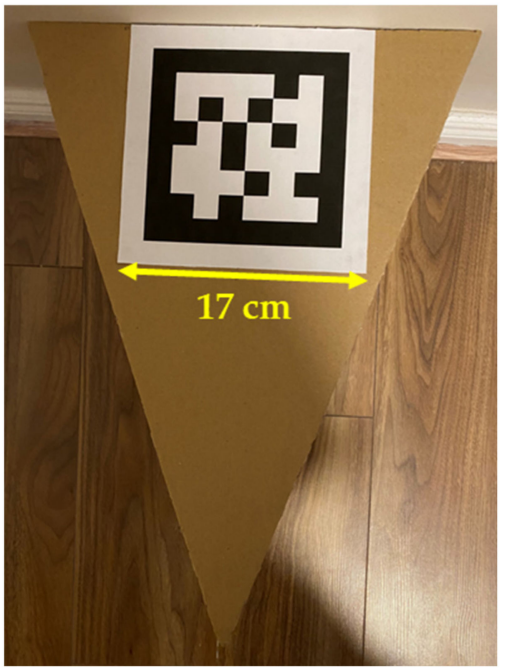

(a)

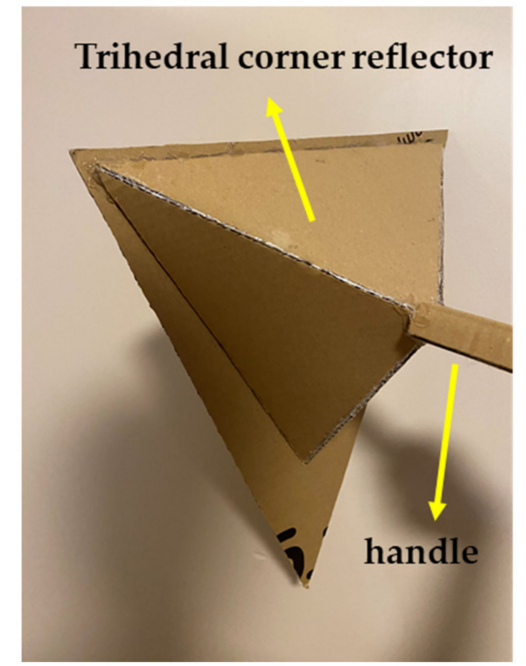

(b)

Figure 11. The proposed triangular calibration target design to spatial temporal calibrates the sensors (camera, radar, LiDAR). (a) Front view of the calibration board consists of a printed AprilTag marker with a size of approximately $17 \mathrm{~cm}$ in length. (b) The trihedral corner reflector is attached at the rear of the triangular board in which the inner sides are overlaid with aluminum foil. The calibration target in figure is constructed based on and reference [169-171] and through personal communication [172].

Other spatial-temporal calibration methods include employing a target-based approach and the spatial-temporal relationships of the target measurements (positions) to estimate the time delays and the sensors extrinsic parameters [173]. In [174], the PolySync bus (external hardware) was employed to publish a synchronized timestamp based on the 
IEEE 1588 Precision Time Protocol (PTP), to all computers as a means of time synchronization during the data acquisition process.

To summarize, estimating the time delays between multiple sensors operating at different frequencies is vital, especially in time-critical autonomous systems, to precisely perform autonomous tasks in real-time, such as obstacles detection, and vehicle state estimation, and ultimately to prevent collisions.

\subsection{Sensor Fusion}

Sensor fusion is an essential aspect of most autonomous systems, e.g., on-road selfdriving cars and autonomous Unmanned Ground Vehicles (UGV). It integrates the acquired data from multiple sensing modalities to reduce the number of detection uncertainties and overcome the shortcomings of individual sensors operating independently. Moreover, sensor fusion helps to develop a consistent model that can perceive the surroundings accurately in various environmental conditions [175]. For instance, camera and radar fusion may provide high-resolution images and the relative velocities of the detected obstacles in the perceived scene. Table 7 below qualitatively summarizes the strengths and weaknesses of the commonly utilized perception-based sensors in AVs based on their technical characteristics and other external factors, such as weather and illumination conditions.

Table 7. A comparison of the commonly employed sensors in self-driving cars; camera, LiDAR, and radar, based on technical characteristics and other external factors. The " $\checkmark$ " symbol indicates that the sensor operates competently under the specific factor. The " $\sim$ " symbol indicates that the sensor performs reasonably well under the specific factor. The " $x$ " symbol indicates that the sensor does not operate well under the specific factor relative to the other sensors.

\begin{tabular}{ccccc}
\hline Factors & Camera & LiDAR & Radar & Fusion \\
\hline Range & $\sim$ & $\sim$ & $\checkmark$ & $\checkmark$ \\
\hline Resolution & $\checkmark$ & $\sim$ & $\checkmark$ & $\checkmark$ \\
\hline Distance Accuracy & $\sim$ & $\checkmark$ & $\times$ & $\checkmark$ \\
\hline Velocity & $\sim$ & $x$ & $\checkmark$ & $\checkmark$ \\
\hline $\begin{array}{c}\text { Color Perception, e.g., } \\
\text { traffic lights }\end{array}$ & $\checkmark$ & $\checkmark$ & $\times$ & $\checkmark$ \\
\hline Object Detection & $\sim$ & $\sim$ & $\checkmark$ \\
\hline Object Classification & $\checkmark$ & $\times$ & $\times$ & $\checkmark$ \\
\hline Lane Detection & $\checkmark$ & $\checkmark$ & $\checkmark$ & $\checkmark$ \\
\hline Obstacle Edge Detection & $\checkmark$ & $\checkmark$ & $\checkmark$ & $\checkmark$ \\
\hline Illumination Conditions & $\times$ & $\sim$ & & $\checkmark$ \\
\hline Weather Conditions & $\times$ & & $\checkmark$ & $\checkmark$ \\
\hline
\end{tabular}

The research on multi-sensor fusion systems in $\mathrm{AVs}$ for environment perception and object detection is well-established in the literature [19,21,30,167,176-178]. Presently, three primary sensor combinations for obstacle detection are prevalent in the literature, including camera-LiDAR (CL); camera-radar (CR); and camera-LiDAR-radar (CLR) sensor combinations. A survey conveyed by [21] showed that the CR sensor combination is the most employed in the multi-sensor fusion systems for environment perception, followed by CLR and $\mathrm{CL}$. The CR sensor combination offers high-resolution images while obtaining additional distance and velocity information of surrounding obstacles. For instance, Tesla utilized the $\mathrm{CR}$ sensor combination and other sensors, such as ultrasonic sensors, to perceive vehicle surroundings [8]. Similarly, the CLR sensor combination can provide resolution at a greater range, and precisely understands the surroundings through the LiDAR point clouds, and depth map information. It also improves the safety redundancy of the overall autonomous 
system. For instance, Waymo and Navya [179] used the CLR sensor combination for environment perception in their AVs.

\subsubsection{Sensor Fusion Approaches}

There are three primary approaches to combine sensory data from various sensing modalities in the MSDF frameworks: high-level fusion (HLF), low-level fusion (LLF), and mid-level fusion (MLF) [180]. In the HLF approach, each sensor carries out object detection or a tracking algorithm independently and subsequently performs fusion. For instance, reference [30] utilized the HLF approach to fuse the processed data, i.e., radar signals and LiDAR point clouds independently and subsequently used a non-linear Kalman Filter method to detect obstacles and state tracking. The HLF approaches are often adopted due to a lower relative complexity than the LLF and MLF approach. However, HLF provides inadequate information as classifications with a lower confidence value are discarded if, for example, there are several overlapping obstacles.

Contrarily, with the LLF approach, data from each sensor are integrated (or fused) at the lowest level of abstraction (raw data). Therefore, all information is retained and can potentially improve the obstacle detection accuracy. Reference [181] proposed a two-stage 3 D obstacle detection architecture, named 3D-cross view fusion (3D-CVF). In the second stage, they utilized the LLF approach to fuse the joint camera-LiDAR feature map obtained from the first stage with the low-level camera and LiDAR features using a $3 D$ region of interest (RoI)-based pooling method. They evaluated the proposed method on KITTI and nuScenes datasets and reported that the object detection results outperformed the state-of-the-art 3D object detectors in the KITTI leaderboard (see reference [181] for a more comprehensive summary). In practice, the LLF approach comes with a multitude of challenges, not least in its implementation. It requires precise extrinsic calibration of sensors to accurately fuse their perceptions of the environment. The sensors must also counterbalance ego-motion (3D motion of a system within an environment) and be temporally calibrated [180].

The MLF, otherwise known as feature-level fusion, is an abstraction level between LLF and HLF. It fuses multi-target features extracted from the corresponding sensor data (raw measurements), such as color information from images or location features of radar and LiDAR, and subsequently perform recognition and classification on the fused multisensor features. Reference [182] proposed a feature-level sensor fusion framework to detect targets in a dynamic background environment with limited communication capability. They utilized the Symbolic Dynamic Filtering (SDF) algorithm to extract the low-dimensional features from multiple infrared sensors in different orientations and in the presence of changing ambient light intensities and subsequently fusing the extracted features as clusters with the agglomerative hierarchical clustering algorithm for moving target detection. The MLF, however, appears to be insufficient to achieve a SAE Level 4 or Level 5 AD system due to its limited sense of the environment and loss of contextual information [183].

\subsubsection{Sensor Fusion Techniques and Algorithms}

Sensor fusion techniques and algorithms have been extensively studied over the last number of years and now, are well-established in the literature. However, a recent study $[184,185]$ revealed that obtaining the current state-of-the-art fusion techniques and algorithms is an arduous and challenging task due to multidisciplinary and variants of proposed fusion algorithms in the literature. The study of [19] classified these techniques and algorithms into classical sensor fusion algorithms and deep learning sensor fusion algorithms. On the one hand, the classical sensor fusion algorithms, such as knowledgebased methods, statistical methods, probabilistic methods, et cetera, utilize the theories of uncertainty from data imperfections, including inaccuracy and uncertainty to fuse sensor data. Reference [186] proposes a real-time roundabout detection and navigation system in a road environment utilizing a combination of the proposed "Laser Simulator" algorithm to detect objects and the knowledge-based fuzzy logic (FL) algorithm for decision making. 
On the other hand, the deep learning sensor fusion algorithms involve generating various multi-layer networks that enable them to process raw data and extract features to perform challenging and intelligent tasks, e.g., object detection in an urban environment for AV. In the AV context, algorithms, such as Convolutional Neural Network (CNN) and Recurrent Neural Network (RNN) are among the most employed algorithms in perception systems. Reference [187] proposed an advanced weighted-mean You Only Look Once (YOLO) CNN algorithms to fuse RGB camera and LiDAR point cloud data to improve the real-time performance of object detection. YOLO detector was first created in 2016 by [188] and has achieved a significant milestone over the last number of years. It is a single-stage detector that predicts bounding boxes and produces class probabilities with confidence scores on an image in a single neural network (one evaluation only). The YOLO based model provides fast detection speed of 45 FPS with $59.2 \%$ average precision (AP, an evaluation metric that measures object detection or information retrieval model performances) on the VOC 2007 dataset [188]. Besides, the latest YOLOv4 released by [189] in April 2020, achieves state-of-the-art results at a real-time speed on the MS COCO dataset of approximately 65 FPS with $43.5 \%$ AP (and $65.7 \%$ AP50-IoU above $50 \%$ ) on an NVIDIA ${ }^{\circledR}$ Tesla ${ }^{\circledR}$ V100 Graphical Processing Unit (GPU). In [190], the authors proposed a CNN-based method to detect aggressive driving behaviors through emotions using near-infrared light and thermal cameras. They conducted score-level fusion using the CNN output scores from near-infrared light images and thermal images to improve the detection accuracy. Their proposed method achieved a high classification accuracy of emotions and demonstrated that their proposed technique achieved better performance than the conventional methods for emotion detection.

In addition, with the advent of 3D sensors and diverse applications for understanding the 3D environment of the surrounding $\mathrm{AV}$, there is an increased research focus on 3D object detection. Reference [191] leverages their previously proposed VoxelNet framework in [192] and presented two feature-level fusion approaches called PointFusion and VoxelFusion to combine the RGB and point cloud data for 3D object detection. According to [192], VoxelNet is a generic 3D object detection network that unifies feature extraction and bounding box prediction processes into a single stage, end-to-end trainable deep network. The PointFusion method uses the known calibration matrix to project 3D points onto the image and, subsequently extracts image features from a pre-trained 2D CNN and concatenate them at the point level. Subsequently, they leveraged the VoxelNet architecture to process the concatenated features and the corresponding points jointly. In contrast, the VoxelFusion method projects the non-empty 3D voxels created by the VoxelNet onto the image and extract features within the 2D ROIs and consequently concatenates the poo-led image features at the voxel level.

Reference [193] presented a PointFusion framework that leverages the image data and raw point cloud data for 3D object detection. They utilized the CNN and PointNet [194] architectures to process the image and point cloud independently and subsequently combine the resulting outputs to predict multiple 3D box hypothesis and their corresponding confidences. The PointNet architecture is a novel neural network that provides a unified architecture for applications ranging from 3D classification to scene semantic parsing for processing raw point cloud data. Other deep learning-based sensor fusion algorithms, to name a few, include:

- ResNet, or Residual Networks, is a residual learning framework that facilitates deep networks training [195].

- SSD, or Single-Shot Multibox Detector, is a method that discretizes bounding boxes into a set of boxes with different sizes and aspect ratios per feature map location to detect objects with variant sizes [196]—it overcomes the limitation of YOLO small and variant-scale object detection accuracy.

- CenterNet [197] represents the state-of-the-art monocular camera 3D object detection algorithm, which leverages key-point estimation to find center points of bounding 
boxes and regresses the center points to all other object properties, including size, 3D location, orientation, and pose.

Table 8a below summarizes the strengths and weaknesses of the sensor fusion approaches: HLF, LLF, and MLF, and presents an overview of the sensor fusion techniques and algorithms for obstacle detection, namely YOLO, SSD, VoxelNet, and PointNet, in Table $8 \mathrm{~b}$. The readers interested in detailed discussions about sensor fusion techniques and algorithms for various applications ranging from perception, including $2 \mathrm{D}$ or 3D obstacle detection and lane tracking, to localization and mapping are advised to refer to $[19,20,23-25,184,191-206]$.

Table 8. (a) A comparative overview of the sensor fusion approaches, namely high-level fusion (HLF), low-level fusion (LLF), and mid-level fusion (MLF) [30,180,204-206]. (b) Table below summarizes some of the sensor fusion techniques and algorithms that were successfully established in the art for obstacles detection, namely YOLO, SSD, VoxelNet, and PointNet. Further, table below presents a summary of the advantages and drawbacks of each algorithm.

(a)

\begin{tabular}{cc}
\hline Sensor Fusion Approaches & \multicolumn{1}{c}{ Descriptions } \\
\hline High-Level Fusion (HLF) & $\begin{array}{l}\text { Each sensor carries out detection } \\
\text { or tracking algorithm separately } \\
\text { and subsequently combines the } \\
\text { result into one global decision. }\end{array}$
\end{tabular}

Lower complexity and requires less computational load and communication resources. Further, HLF enables standardizing the interface towards the fusion algorithm and does not necessitate an in-depth understanding of the signal processing algorithms involved.

Sensor information is retained and provides more accurate data (a lower signal-to-noise ratio) than the individual sensors operating independently. As a result, it has the potential to improve the detection accuracy. In addition, LLF reduces latency where the domain controller does not have to wait for the sensor to process the data before acting upon it. This can help to speed up the performance-of particular importance in time-critical systems.

ore integrated data) to be of better quality and more informative.
Extracts contextual descriptions or features from each sensor data (raw measurements) and

Mid-Level Fusion (MLF) subsequently fuses the features from each sensor to produce a fused signal for further processing.
Generates small information spaces and requires less computation load approach provides a powerful feature vector and the features selection algorithms that detect corresponding features and features subsets can improve the recognition accuracy. than LLF approaches. Further, MLF

Provides inadequate information as classifications with a lower confidence value are discarded. Furthermore, fine-tuning the fusion algorithms has a negligible impact on the data accuracy or latency.

Generates large amount of data that could be an issue in terms of memory or communication

bandwidth. Further, LLF requires precise calibration of sensors to accurately fuse their perceptions and it may pose a challenge to handle incomplete measurements. Although multi-source data can be fused to the maximum extent, there is data redundancy, which results in low fusion efficiency.

Requires large training sets to find the most significant feature subset. It requires precise sensor calibration before extracting and fusing the features from each sensor.

(b)

\begin{tabular}{cc}
\hline Algorithms & Descriptions \\
\hline & \\
& You Only Look Once (YOLO) is a \\
single-stage detector, which \\
predicts bounding boxes and \\
produces class probabilities with \\
confidence scores on an image in \\
a single CNN ${ }^{1}$.
\end{tabular}

Reference

Provides real-time detections.

Less accurate than SSD.

Poor detection of dense obstacles, e.g., flocks of birds, because each grid can propose only 2 bounding boxes.

- Poor detection of small obstacles.

High localization error. 
Table 8. Cont.

\begin{tabular}{|c|c|c|c|}
\hline Sensor Fusion Approaches & Descriptions & Strengths & Weaknesses \\
\hline SSD & $\begin{array}{l}\text { Single-Shot Multibox Detector } \\
\text { (SSD) is a single-stage CNN } \\
\text { detector that discretizes bounding } \\
\text { boxes into a set of boxes with } \\
\text { different sizes and aspect ratios to } \\
\text { detection obstacles with variant } \\
\text { sizes. }\end{array}$ & 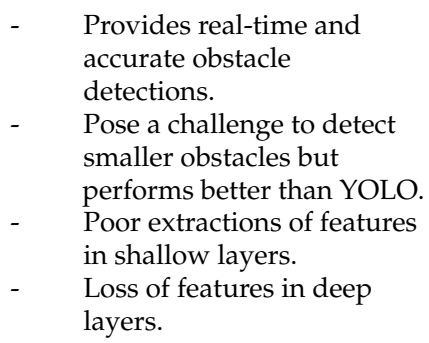 & {$[19,196,200]$} \\
\hline VoxelNet & $\begin{array}{l}\text { A generic 3D obstacle detection } \\
\text { network that unifies feature } \\
\text { extraction and bounding boxes } \\
\text { prediction into a single-stage, } \\
\text { end-to-end trainable deep } \\
\text { network. In other words, } \\
\text { VoxelNet is a voxelized method } \\
\text { for obstacle detection using point } \\
\text { cloud data. }\end{array}$ & $\begin{array}{l}\text { - Does not require to extract } \\
\text { features manually. } \\
\text { Requires large volume of } \\
\text { data and memory for } \\
\text { training. }\end{array}$ & {$[192,202]$} \\
\hline PointNet & $\begin{array}{c}\text { Presents a permutation-invariant } \\
\text { deep neural network which learns } \\
\text { global features from unordered } \\
\text { point clouds (two-stage } \\
\text { detection). }\end{array}$ & $\begin{array}{l}\text { - Able to handle point clouds } \\
\text { in any order, e.g., } \\
\text { permutation independence } \\
\text { on the order of point } \\
\text { clouds. } \\
\text { Difficult to generalize to } \\
\text { unseen point } \\
\text { configurations. }\end{array}$ & {$[194,202]$} \\
\hline
\end{tabular}

\footnotetext{
${ }^{1} \mathrm{CNN}$, or Convolutional Neural Network, is a specialized neural network that is used to process data that has an input shape like a $2 \mathrm{D}$ matrix, such as images.
}

\subsubsection{Challenges of Sensor Fusion for Safe and Reliable Environment Perception}

Undoubtedly, the multi-sensor fusion technologies, based on extensive research, have achieved relatively comprehensive advantages in autonomous systems ranging from humanoid robots to AVs. These systems are often equipped with an array of sensors that could generate a large volume of data per hour. For instance, an AV could generate approximately 383 GB to 5.17 TB (Terabyte) of data per hour [207]. Therefore, it requires large computational power to process these data. Reference [208] reviewed the computing platform implementation of an SAE Level $4 \mathrm{AV}$ from a leading autonomous driving company and examined several existing processing solutions for $\mathrm{AD}$. In addition, they presented and prototyped an $\mathrm{AD}$ computing architecture and software stack that is secure, modular, dynamic, energy-efficient, and high performance. Their prototype system consumes an average of 11 Watt $(\mathrm{W})$ of power and can drive a mobile vehicle at $8 \mathrm{~km}$ per hour, using an ARM Mobile System on Chip (SoC). From the software perspective, combining reinforcement learning (RL) techniques with supervised learning algorithms could help to reduce computational power, training data requirements, and training time.

$\mathrm{RL}$ is a machine learning (ML) method that uses the feedback from their actions and experiences to train ML models in an interactive environment. In contrast, supervised learning algorithm utilizes labelled data to train ML models (refer to reference [25] for a more detailed overview). However, it is challenging to train and annotate data from all possible scenarios, including but not limited to location, terrain, and weather, which an AV may encounter in the real-world. Although collaboration and sharing of data could benefit the development of autonomous systems, it is unlikely as companies researching autonomous systems are unwilling to share resources due to the fear of diluting their competitive advantage $[25,209]$. Additionally, the performance of an ML/DL for object detection and localization and mapping are influenced by the employed dataset's quality; 
hence, poor data quality could lead to the proverbial "garbage-(data)-in and garbage-(data)out". The founder and CTO of Roboflow wrote that 33\% out of 15,000 samples (or images) in Udacity Dataset 2 are not annotated and the annotated bounding boxes (or objects of interest) are oversize [210].

The functional safety of the utilized DL models in multi-sensor AVs can also be a challenge due to the opaque nature of DL algorithms. Reference [25] highlighted that it is critical to further research the available safety validation methods and the interpretability of neural networks before deploying DL models on the road. In addition, autonomous systems that utilize DL architectures are vulnerable to adversarial attacks. The attackers overlaid typical images with adversarial samples (or perturbed images) that represent subtle changes to the inputs of the DL systems but resulted in misclassification of objects with high confidence scores [25]. Other sensor fusion challenges include biases in collected datasets, overfitting of training datasets, imprecision, and uncertainty in the data measurements, such as noise relating to calibration errors, quantization errors, loss of precisions, missing values, et cetera. Transforming multi-sensor data into a standard frame of reference may also pose a challenge in sensor fusion implementations.

From an environmental perspective, one of the remaining challenges of sensor fusion for reliable and safe perception is the performance of vision sensors in harsh weather conditions such as snow, fog, sandstorms, or rainstorm. Such conditions can impact the vision and range measurements of vision sensors, leading to a decrease in visibility distance and resulting in erroneous and misleading outputs. In a worst-case scenario, sensors may experience a partial or complete sensor failure, which can be disastrous for AVs and their surroundings. Hence, based on learned experiences and historical data, it is important to evaluate the risk of failure early in the process and enable drivers to interrupt or completely disengage the autonomous systems [19].

In general, quality data is the key to a safe and reliable environment perception. DL/ML models employ these data to learn about the environment's features and perform object detection. Thus, it is essential to cleanse and pre-process the data before implementing DL/ML algorithms. However, DL algorithms are prone to malicious attacks, which can be disastrous in safety-critical systems, such as AVs. Further research and extensive testing of autonomous systems are essential to assess all possible solutions to prevent malicious attacks and evaluate all possible sensors and system failure risks and alternative solutions in the case of sensors or system failures. A detailed discussion about the sensor fusion challenges, including adversarial attacks and possible preventions is beyond the scope of this paper (see $[16,19,25,211-214]$ for a more comprehensive overview).

\section{Conclusions and Future Research Recommendations}

In this paper, we presented a complete overview of the perception block in the $\mathrm{AD}$ systems. We surveyed the technical performance and capabilities of sensors from various manufacturers in different conditions, mainly focusing on vision cameras, LiDAR sensors, and radar sensors. We also presented an overview of the three main categories of sensor calibration, which may be considered a foundation block of any autonomous systems and summarize the existing open-source multi-sensor calibration packages that can calibrate multiple sensors simultaneously. Finally, we reviewed some of the fusion algorithms that were successfully established in the literature and highlighted some of the challenges in the sensor fusion field and possible future research directions for AD systems.

The area of AVs is vast and consists of a wide range of technical disciplines and technologies, from electronics, sensors, and hardware to algorithms for vehicle state control and decision-making, and economic, legal, and social aspects. Sensors are elementary to the perception of surroundings, localization and mapping, and vehicle state control. Currently, AVs primarily incorporate multiple, complementary sensors, such as IMUs, radars, LiDARs, and cameras to overcome the limitations of individual sensors operating independently.

It is essential to calibrate sensors before the implementation of algorithms for processing data. A precise sensor calibration allows the AV to understand its position and 
orientation in the real-world coordinates. We examined the three main categories of sensor calibration, each of which is necessary: namely, intrinsic calibration, extrinsic calibration, and temporal calibration and related algorithms. Additionally, we provided a comparative overview of several existing open-source calibration packages that have been successfully employed in recent research. It is apparent that most existing open-source calibration tools for extrinsic and temporal calibration only address pairwise calibration of a maximum of two sensing modalities.

The approaches to sensor calibration in recent studies focus on offline methods to calibrate the sensors. The offline method to sensor calibration utilizes the specially designed calibration targets to provide accurate calibration results, but it is not flexible. For instance, the vehicle is required to recalibrate if there is a geometry change between the sensors. Moreover, external factors, such as temperature and vibrations, may affect the calibration accuracy as multi-sensor are commonly factory calibrated. Therefore, it is critical to further research online and offline calibration techniques to automatically detect and refine calibration parameters to provide precise estimation of the presence and position of objects in autonomous operation.

The development of reliable and efficient obstacle detection in self-driving vehicles is critical to achieving autonomous driving. The practical approach in recent studies for safe and reliable obstacle detection is to combine information from multimodal sensors, such as distance information, velocity, color distribution, et cetera, to provide accurate, robust, and reliable detection results. We reviewed the three primary approaches of sensor fusion: namely high-level fusion, mid-level fusion, and low-level fusion and subsequently reviewed recently proposed multi-sensor fusion techniques and algorithms for obstacle detection. Similarly, we highlighted several challenges of multi-sensor fusion for reliable and safe environment perception. The main challenges are environmental conditions, invulnerability to malicious attacks in DL models, poor quality datasets, or datasets not addressing all possible environments for the $\mathrm{AV}$, and the computation cost to process large volumes of datasets in real-time. Therefore, companies and researchers must evaluate the risk of failure and implement alternative solutions for drivers to handle worst-case scenarios.

Further developments to improve object detection performance in all possible scenarios, including harsh weather conditions, are essential to providing safe and reliable scene perception. It is critical to developing accurate, robust, and reliable object detection algorithms that can distinguish obstacles against the environment. One approach to providing a more reliable and accurate obstacle detection is to enhance existing sensor fusion algorithms through deep learning approaches or deep reinforcement learning approaches [215]. Another approach would be to invest in sensors hardware technology to provide a higher resolution of the surroundings [19].

Author Contributions: Conceptualization, D.J.Y;; Methodology, D.J.Y. and G.V.-H.; Software, D.J.Y.; Validation, D.J.Y., G.V.-H. and J.B.; Formal analysis, D.J.Y.; Investigation, D.J.Y. and G.V.-H.; Resources, D.J.Y.; Data curation, D.J.Y.; Writing—original draft preparation, D.J.Y.; Writing-review and editing, D.J.Y., G.V.-H., and J.B.; Visualization, D.J.Y.; Supervision, J.B. and J.W.; Project Administration, D.J.Y., G.V.-H., J.B., and J.W.; Funding Acquisition, J.B. and J.W. All authors have read and agreed to the published version of the manuscript.

Funding: This research reported in this review article was supported, in part, by the Science Foundation Ireland grant 13/RC/2094_P2 and co-funded under the European Regional Development Fund through the Southern \& Eastern Regional Operational Programme to Lero-the Science Foundation Ireland Research Centre for Software (www.lero.ie).

Institutional Review Board Statement: Not applicable.

Informed Consent Statement: Not applicable.

Data Availability Statement: No new data were created or analyzed in this study. Data sharing is not applicable to this article. 
Acknowledgments: This paper and research behind it would not have been possible without the support of the IMaR team in the Munster Technological University.

Conflicts of Interest: The authors declare no conflict of interest.

\section{References}

1. World Health Organization. Global Status Report on Road Safety; WHO: Geneva, Switzerland, 2018; ISBN 978-9241565684.

2. Road I Mobility and Transport. Available online: https://ec.europa.eu/transport/themes/its/road_it (accessed on 20 November 2020).

3. Autonomous Vehicle Market to Garner Growth 63.5\%. Available online: https://www.precedenceresearch.com/autonomousvehicle-market (accessed on 19 November 2020).

4. Glon, R.; Edelstein, S. The History of Self-Driving Cars. 2020. Available online: https:/ /www.digitaltrends.com/cars/history-ofself-driving-cars-milestones / (accessed on 18 November 2020).

5. Wiggers, K. Waymo's Autonomous Cars Have Driven 20 Million Miles on Public Roads. 2020. Available online: https: / /venturebeat.com/2020/01/06/waymos-autonomous-cars-have-driven-20-million-miles-on-public-roads / (accessed on 18 November 2020).

6. Jaguar Land Rover to Partner with Autonomous Car Hub in Shannon. 2020. Available online: https://www.irishtimes.com/ business / transport-and-tourism/jaguar-land-rover-to-partner-with-autonomous-car-hub-in-shannon-1.4409884 (accessed on 25 November 2020).

7. Shuttleworth, J. SAE Standard News: J3016 Automated-Driving Graphic Update. 2019. Available online: https://www.sae.org/ news/2019/01/sae-updates-j3016-automated-driving-graphic (accessed on 18 November 2020).

8. Autopilot. Available online: https://www.tesla.com/en_IE/autopilot (accessed on 23 November 2020).

9. Footage Audi A8: Audi AI Traffic Jam Pilot. Available online: https://www.audi-mediacenter.com/en/audimediatv/video/ footage-audi-a8-audi-ai-traffic-jam-pilot-3785\#: \{\}:text=The \%20Audi\%20AI\%20traffic $\% 20$ jam, \%2Fh\%20(37.3\%20mph) (accessed on 23 November 2020).

10. Edelstein, S. Audi Gives up on Level 3 Autonomous Driver-Assist System in A8. 2020. Available online: https: / / www.motorauthority.com/news/1127984_audi-gives-up-on-level-3-autonomous-driver-assist-system-in-a8 (accessed on 23 November 2020).

11. Sage, A. Waymo Unveils Self-Driving Taxi Service in Arizona for Paying Customers. 2018. Available online: https: / / www.reuters.com/article/us-waymo-selfdriving-focus / waymo-unveils-self-driving-taxi-service-in-arizona-for-payingcustomers-idUSKBN1O41M2 (accessed on 23 November 2020).

12. Mozaffari, S.; Al-Jarrah, O.Y.; Dianati, M.; Jennings, P.; Mouzakitis, A. Deep Learning-Based Vehicle Behavior Prediction for Autonomous Driving Applications: A Review. IEEE Trans. Intell. Transp. Syst. 2020, 1-15. [CrossRef]

13. Mehra, A.; Mandal, M.; Narang, P.; Chamola, V. ReViewNet: A Fast and Resource Optimized Network for Enabling Safe Autonomous Driving in Hazy Weather Conditions. IEEE Trans. Intell. Transp. Syst. 2020, 1-11. [CrossRef]

14. Gonzalez-de-Santos, P.; Fernández, R.; Sepúlveda, D.; Navas, E.; Emmi, L.; Armada, M. Field Robots for Intelligent FarmsInhering Features from Industry. Agronomy 2020, 10, 1638. [CrossRef]

15. Velasco-Hernandez, G.; Yeong, D.J.; Barry, J.; Walsh, J. Autonomous Driving Architectures, Perception and Data Fusion: A Review. In Proceedings of the 2020 IEEE 16th International Conference on Intelligent Computer Communication and Processing (ICCP 2020), Cluj-Napoca, Romania, 3-5 September 2020.

16. Giacalone, J.; Bourgeois, L.; Ancora, A. Challenges in aggregation of heterogeneous sensors of Autonomous Driving Systems. In Proceedings of the 2019 IEEE Sensors Applications Symposium (SAS), Sophia Antipolis, France, 11-13 March 2019.

17. Liu, X.; Baiocchi, O. A comparison of the definitions for smart sensors, smart objects and Things in IoT. In Proceedings of the 2016 IEEE 7th Annual Information Technology, Electronics and Mobile Communication Conference (IEMCON), Vancouver, BC, Canada, 13-15 October 2016.

18. Wojciechowicz, T. Smart Sensor vs Base Sensor-What's the Difference? I Symmetry Blog. Available online: https: / / www.semiconductorstore.com/blog/2018/Smart-Sensor-vs-Base-Sensor-Whats-the-Difference-Symmetry-Blog/3538/\#: $\sim\{\}:$ text=By\%20using\%20a\%20smart\%20sensor,achieve\%20on\%20a\%20base\%20sensor (accessed on 26 November 2020).

19. Fayyad, J.; Jaradat, M.A.; Gruyer, D.; Najjaran, H. Deep Learning Sensor Fusion for Autonomous Vehicle Perception and Localization: A Review. Sensors 2020, 20, 4220. [CrossRef] [PubMed]

20. Campbell, S.; O’Mahony, N.; Krpalcova, L.; Riordan, D.; Walsh, J.; Murphy, A.; Conor, R. Sensor Technology in Autonomous Vehicles: A review. In Proceedings of the 2018 29th Irish Signals and Systems Conference (ISSC), Belfast, UK, $21-22$ June 2018.

21. Wang, Z.; Wu, Y.; Niu, Q. Multi-Sensor Fusion in Automated Driving: A Survey. IEEE Access 2019, 8, 2847-2868. [CrossRef]

22. Yeong, D.J.; Barry, J.; Walsh, J. A Review of Multi-Sensor Fusion System for Large Heavy Vehicles Off Road in Industrial Environments. In Proceedings of the 2020 31st Irish Signals and Systems Conference (ISSC), Letterkenny, Ireland, 11-12 June 2020.

23. Jusoh, S.; Almajali, S. A Systematic Review on Fusion Techniques and Approaches Used in Applications. IEEE Access 2020, 8, 14424-14439. [CrossRef]

24. Castanedo, F. A Review of Data Fusion Techniques. Sci. World J. 2013, 2013, 19. [CrossRef]

25. Kuutti, S.; Bowden, R.; Jin, Y.; Barber, P.; Fallah, S. A Survey of Deep Learning Applications to Autonomous Vehicle Control. IEEE Trans. Intell. Transp. Syst. 2021, 22, 712-733. [CrossRef] 
26. Hu, J.-W.; Zheng, B.-Y.; Wang, C.; Zhao, C.-H.; Hou, X.-L.; Pan, Q.; Xu, Z. A Survey on multi-sensor fusion based obstacle detection for intelligent ground vehicles in off-road environments. Front. Inform. Technol. Electron. Eng. 2020, 21, 675-692. [CrossRef]

27. Mobile Robot Sensors. Available online: http://www.robotiksistem.com/robot_sensors.html (accessed on 24 November 2020).

28. Robotic Autonomy Summer Camp. Available online: http://www.cs.cmu.edu/ \{\}rasc/Download/AMRobots4.pdf (accessed on 24 November 2020).

29. Woo, A.; Fidan, B.; Melek, W.W. Localization for Autonomous Driving. In Handbook of Position Location: Theory, Practice, and Advances, 2nd ed.; Zekavat, S., Buehrer, R.M., Eds.; Wiley-IEEE Press: Hoboken, NJ, USA, 2019; pp. 1051-1087. ISBN 978-1-119-43458-0.

30. Shahian Jahromi, B.; Tulabandhula, T.; Cetin, S. Real-Time Hybrid Multi-Sensor Fusion Framework for Perception in Autonomous Vehicles. Sensors 2019, 19, 4357. [CrossRef]

31. Guo, X. Feature-Based Localization Methods for Autonomous Vehicles. Ph.D. Thesis, Freien Universität Berlin, Berlin, Germany, 2017.

32. Wendt, Z.; Jeremy Cook, S. Saved by the Sensor: Vehicle Awareness in the Self-Driving Age. 2019. Available online: https:/ / www.machinedesign.com/mechanical-motion-systems/article/21836344/saved-by-the-sensor-vehicle-awareness-inthe-selfdriving-age (accessed on 25 November 2020).

33. Joglekar, A.; Joshi, D.; Khemani, R.; Nair, S.; Sahare, S. Depth Estimation Using Monocular Camera. IJCSIT 2011, 2, $1758-1763$.

34. Bhoi, A. Monocular Depth Estimation: A Survey. arXiv 2019, arXiv:1901.09402v1.

35. Garg, R.; Wadhwa, N.; Ansari, S.; Barron, J.T. Learning Single Camera Depth Estimation using Dual-Pixels. arXiv 2019, arXiv:1904.05822v3.

36. Cronin, C.; Conway, A.; Walsh, J. State-of-the-Art Review of Autonomous Intelligent Vehicles (AIV) Technologies for the Automotive and Manufacturing Industry. In Proceedings of the 2019 30th Irish Signals and System Conference (ISSC), Maynooth, Ireland, 17-18 June 2019.

37. Orbbec-Intelligent computing for everyone everywhere. Available online: https:/ / orbbec3d.com/ (accessed on 4 December 2020).

38. Harapanahalli, S.; O’Mahony, N.; Velasco-Hernandez, G.; Campbell, S.; Riordan, D.; Walsh, J. Autonomous Navigation of mobile robots in factory environment. Procedia Manuf. 2019, 38, 1524-1531. [CrossRef]

39. Stereo_Image_Proc-ROS Wiki. Available online: http://wiki.ros.org/stereo_image_proc (accessed on 4 December 2020).

40. 3D Camera Survey-ROS-Industrial. Available online: https://rosindustrial.org/news/2016/1/13/3d-camera-survey (accessed on 23 November 2020).

41. Roboception 3D Stereo Sensor. Available online: https://roboception.com/wp-content/uploads/2020/06/202006_3D_ StereoSensor.pdf (accessed on 23 November 2020).

42. MultiSense S7-Carnegie Robotics LLC. Available online: https:/ / carnegierobotics.com/multisense-s7 (accessed on 23 November 2020).

43. Knabe, C.; Griffin, R.; Burton, J.; Cantor-Cooke, G.; Dantanarayana, L.; Day, G.; Ebeling-Koning, O.; Hahn, E.; Hopkins, M.; Neal, J.; et al. Team VALOR's ESCHER: A Novel Electromechanical Biped for the DARPA Robotics Challenge. J. Field Robot. 2017, 34, 1-27. [CrossRef]

44. MultiSense S21B-Carnegie Robotics LLC. Available online: https://carnegierobotics.com/multisense-s21b (accessed on 23 November 2020).

45. N-Series Model Listing I Ensenso. Available online: https://www.ensenso.com/support/modellisting/?id=N35-606-16-BL (accessed on 24 November 2020).

46. FRAMOS Industrial Depth Camera D435e-Starter Kit I FRAMOS. Available online: https://www.framos.com/en/framosdepth-camera-d435e-starter-kit-22805 (accessed on 25 November 2020).

47. Karmin 3D Stereo Camera-Nerian Vision Technologies. Available online: https://nerian.com/products/karmin3-3d-stereocamera/ (accessed on 26 November 2020).

48. Compare Intel RealSense Depth Cameras (Tech specs and Review). Available online: https://www.intelrealsense.com/comparedepth-cameras / (accessed on 27 November 2020).

49. Bumblebee ${ }^{\circledR 2}$ FireWire I FLIR Systems. Available online: https://www.flir.eu/support/products/bumblebee2-firewire/ \#Overview (accessed on 27 November 2020).

50. Bumblebee ${ }^{\circledR X B 3}$ FireWire I FLIR Systems. Available online: https://www.flir.eu/support/products/bumblebee-xb3-firewire/ \#Overview (accessed on 27 November 2020).

51. Rosero, L.A.; Osório, F.S. Calibration and multi-sensor fusion for on-road obstacle detection. In Proceedings of the 2017 Latin American Robotics Symposium (LARS) and 2017 Brazilian Symposium on Robotics (SBR), Curitiba, Brazil, 8-11 November 2017.

52. Yahiaoui, M.; Rashed, H.; Mariotti, L.; Sistu, G.; Clancy, I.; Yahiaoui, L.; Yogamani, S. FisheyeMODNet: Moving Object Detection on Surround-view Cameras for Autonomous Driving. In Proceedings of the IMVIP 2019: Irish Machine Vision \& Image Processing, Technological University Dublin, Dublin, Ireland, 28-30 August 2019. [CrossRef]

53. Yogamani, S.; Hughes, C.; Horgan, J.; Sistu, G.; Varley, P.; O’Dea, D.; Uricar, M.; Milz, S.; Simon, M.; Amende, K.; et al. WoodScape: A multi-task, multi-camera fisheye dataset for autonomous driving. arXiv 2019, arXiv:1905.01489v2. 
54. Heng, L.; Choi, B.; Cui, Z.; Geppert, M.; Hu, S.; Kuan, B.; Liu, P.; Nguyen, R.; Yeo, Y.C.; Geiger, A.; et al. Project AutoVision: Localization and 3D Scene Perception for an Autonomous Vehicle with a Multi-Camera System. arXiv 2019, arXiv:1809.05477v2.

55. O'Mahony, C.; Campbell, S.; Krpalkova, L.; Riordan, D.; Walsh, J.; Murphy, A.; Ryan, C. Computer Vision for 3D Perception A review. In Proceedings of the 2018 Intelligent Systems Conference (IntelliSys), London, UK, 6-7 September 2018.

56. Petit, F. The Beginnings of LiDAR-A Time Travel Back in History. Available online: https://www.blickfeld.com/blog/thebeginnings-of-lidar/\#: \{\}:text=Lidar\%20technology\%20emerged\%20already\%20in,such\%20as\%20autonomous\%20driving\% 20today (accessed on 20 December 2020).

57. The Automotive LiDAR Market. Available online: http://www.woodsidecap.com/wp-content/uploads/2018/04/Yole_WCPLiDAR-Report_April-2018-FINAL.pdf (accessed on 15 December 2020).

58. A Guide to Lidar Wavelengths. Available online: https:/ / velodynelidar.com/blog/guide-to-lidar-wavelengths / (accessed on 15 December 2020).

59. Wojtanowski, J.; Zygmunt, M.; Kaszczuk, M.; Mierczyk, Z.; Muzal, M. Comparison of 905nm and 1550nm semiconductor laser rangefinders' performance deterioration due to adverse environmental conditions. Opto-Electron. Rev. 2014, 22, 183-190. [CrossRef]

60. Kutila, M.; Pyykönen, P.; Ritter, W.; Sawade, O.; Schäufele, B. Automotive LIDAR sensor development scenarios for harsh weather conditions. In Proceedings of the 2016 IEEE 19th International Conference on Intelligent Transportation Systems (ITSC), Rio de Janeiro, Brazil, 1-4 November 2016.

61. What is LiDAR Technology? Available online: https://blog.generationrobots.com/en/what-is-lidar-technology/\#: \{\}: text=For\%20a\%202D\%20LiDAR\%20only, on \%20X\%20and\%20Y\%20axes.\&text=For\%20a\%203D\%20LiDAR\%2C \%20the,X\%2C\% 20Y\%20and\%20Z\%20axes (accessed on 17 December 2020).

62. Kodors, S. Point Distribution as True Quality of LiDAR Point Cloud. Balt. J. Mod. Comput. 2017, 5, 362-378. [CrossRef]

63. Royo, S.; Ballesta-Garcia, M. An Overview of Lidar Imaging Systems for Autonomous Vehicles. Appl. Sci. 2019, 9, 4093. [CrossRef]

64. Carballo, A.; Lambert, J.; Monrroy-Cano, A.; Wong, D.R.; Narksri, P.; Kitsukawa, Y.; Takeuchi, E.; Kato, S.; Takeda, K. LIBRE: The Multiple 3D LiDAR Dataset. arXiv 2020, arXiv:2003.06129v2.

65. LIBRE: LiDAR Benchmark Reference dataset. Available online: https://sites.google.com/g.sp.m.is.nagoya-u.ac.jp/libre-dataset (accessed on 23 December 2020).

66. Zhao, X.; Yang, Z.; Schwertfeger, S. Mapping with Reflection-Detection and Utilization of Reflection in 3D Lidar Scans. In Proceedings of the 2020 IEEE International Symposium on Safety, Security, and Rescue Robotics (SSRR), Abu Dhabi, United Arab Emirates, 4-6 November 2020.

67. Velodyne-ROS Wiki. Available online: http:/ / wiki.ros.org/velodyne (accessed on 28 December 2020).

68. Products I AutonomouStuff. Available online: https://autonomoustuff.com/products?para1=LiDAR\%20Laser\%20Scanners\& para2=0\&para3=Velodyne (accessed on 28 December 2020).

69. Sualeh, M.; Kim, G.-W. Dynamic Multi-LiDAR Based Multiple Object Detection and Tracking. Sensors 2019, 19, 1474. [CrossRef] [PubMed]

70. Herzog, M.; Dietmayer, K. Training a Fast Object Detector for LiDAR Range Images Using Labeled Data from Sensors with Higher Resolution. In Proceedings of the 2019 IEEE Intelligent Transportation Systems Conference (ITSC), Auckland, New Zealand, 27-30 October 2019.

71. HesaiTechnology/HesaiLidar_General_ROS: ROS driver for PandarXT PandarQT Pandar64 Pandar40P Pandar40M Pandar20A Pandar20B. Available online: https:/ / github.com/HesaiTechnology/HesaiLidar_General_ROS (accessed on 28 December 2020).

72. Pandar64-HESAI. Available online: https://www.hesaitech.com/en/Pandar64 (accessed on 28 December 2020).

73. Pandar40-HESAI. Available online: https://www.hesaitech.com/en/Pandar40 (accessed on 28 December 2020).

74. Ouster-Lidar/Ouster_Example: Ouster Sample Code. Available online: https://github.com/ouster-lidar/ouster_example (accessed on 28 December 2020).

75. OS1 (Serial Number Beginning with “os1-“) Mid-Range High Resolution Imaging Lidar. Available online: http:/ / data.ouster.io/ downloads/OS1-gen1-lidar-sensor-datasheet.pdf (accessed on 28 December 2020).

76. Muckenhuber, S.; Holzer, H.; Bockaj, Z. Automotive Lidar Modelling Approach Based on Material Proper-ties and Lidar Capabilities. Sensors 2020, 20, 3309. [CrossRef]

77. RoboSense-LiDAR/ros_Rslidar: ROS driver for RS-LiDAR-16 and RS-LiDAR-32. Available online: https://github.com/ RoboSense-LiDAR/ros_rslidar (accessed on 28 December 2020).

78. RS-LiDAR-32-RoboSense LiDAR-Autonomous Vehicles, Robots, V2R. Available online: http://www.robosense.ai/en/rslidar/ RS-LiDAR-32 (accessed on 28 December 2020).

79. LSC32/lslidar_c32 at Master Leishen-Lidar/LSC32. Available online: https://github.com/leishen-lidar/LSC32/tree/master/ 1slidar_c32 (accessed on 28 December 2020).

80. LSC16/lslidar_c16 at Master Leishen-Lidar/LSC32. Available online: https:/ / github.com/leishen-lidar/LSC16/tree/master/ lslidar_c16 (accessed on 28 December 2020).

81. 32-Channel LiDAR C32-LeiShenLiDAR/Laser Scanner. Available online: http://www.lslidar.com/product/leida/MX/768ea2 7b-22d2-46eb-9c5d-e81425ef6f11.html (accessed on 28 December 2020).

82. Leishen lslidar-C16 16 channels lidar-Autoware-ROS Discourse. Available online: https://discourse.ros.org/t/leishen-lslidarc16-16-channels-lidar/10055 (accessed on 28 December 2020). 
83. hokuyo3-ROS Wiki. Available online: http://wiki.ros.org/hokuyo3d (accessed on 30 October 2020).

84. Scanning Rangefinder Distance Data Output/YVT-35LX Product Details I HOKUYO AUTOMATIC CO., LTD. Available online: https: / / www.hokuyo-aut.jp/search/single.php?serial=224 (accessed on 30 October 2020).

85. Sick_Ldmrs_Laser_ROS Wiki. Available online: http://wiki.ros.org/sick_ldmrs_laser (accessed on 28 October 2020).

86. Ibeo Standard Four Layer Multi-Echo LUX Sensor I AutonomouStuff. Available online: https://autonomoustuff.com/products/ ibeo-lux-standard (accessed on 28 October 2020).

87. Ibeo Standard Eight Layer/Multi-Echo LUX Sensor I AutonomouStuff. Available online: https://autonomoustuff.com/products / ibeo-lux-81 (accessed on 28 October 2020).

88. DATA SHEET ibeo LUX 4L / ibeo LUX 8L / ibeo LUX HD. Available online: https:/ / hexagondownloads.blob.core.windows.net/ public/AutonomouStuff/wp-content/uploads/2019/05/ibeo_LUX_datasheet_whitelabel.pdf (accessed on 28 October 2020).

89. LD-MRS LD-MRS400102S01 HD, Online Data Sheet. Available online: https://hexagondownloads.blob.core.windows.net/ public/AutonomouStuff/wp-content/uploads/2019/05/LD-MRS400102S01-HD_1052961_en-compressed.pdf (accessed on 29 October 2020).

90. LD-MRS LD-MRS800001S01, Online Data Sheet. Available online: https://hexagondownloads.blob.core.windows.net/public/ AutonomouStuff/wp-content/uploads/2019/05/LD-MRS800001S01_1069408_en-Branded.pdf (accessed on 29 October 2020).

91. Ceptontech/Cepton_sdk_Redist: Cepton SDK Redistribution Channel. Available online: https://github.com/ceptontech/ cepton_sdk_redist (accessed on 12 November 2020).

92. Cepton I Products. Available online: https://www.cepton.com/products.html (accessed on 12 November 2020).

93. Cepton Vista ${ }^{\mathrm{TM}}$-Edge Smart Lidar for Smart Security. Available online: https://www.cepton.com/downloads/Vista-Edgeproduct-brief_0904.pdf (accessed on 12 November 2020).

94. Cepton I Vista®-X90. Available online: https://www.cepton.com/vista-X90.html (accessed on 12 November 2020).

95. Jia, Y.; Guo, L.; Xin, W. Real-time control systems. In Transportation Cyber-Physical Systems, 1st ed.; Deka, L., Chowdhury, M., Eds.; Elsevier: Amsterdam, The Netherlands, 2018; pp. 81-113.

96. Radartutorial. Available online: https://www.radartutorial.eu/11.coherent/co06.en.html (accessed on 28 December 2020).

97. Radar Systems_Doppler Effect-Tutorialspoint. Available online: https://www.tutorialspoint.com/radar_systems/radar_ systems_doppler_effect.htm (accessed on 28 December 2020).

98. Detecting Static Objects in View Using-Electrical Engineering Stack Exchange. Available online: https://electronics. stackexchange.com/questions/236484/detecting-static-objects-in-view-using-radar (accessed on 29 December 2020).

99. Determining the Mounting Position of Automotive Radar Sensors I Rohde \& Schwarz. Available online: https:/ / www.rohdeschwarz.com/applications / determining-the-mounting-position-of-automotive-radarsensors-application-card_56279-661795 .html (accessed on 28 December 2020).

100. Walling, D.H. The Design of an Autonomous Vehicle Research Platform. Master's Thesis, Virginia Polytechnic Institute and State University, Blacksburg, VA, USA, 3 August 2017.

101. Astuff/Astuff_Sensor_Msgs: A Set of Messages Specific to Each Sensor Supported by AutonomouStuff. Available online: https://github.com/astuff/astuff_sensor_msgs/tree/master (accessed on 13 November 2020).

102. Unizg-fer-Lamor / Radar_Interface-Bitbucket. Available online: https://bitbucket.org/unizg-fer-lamor/radar_interface/src/ master/ (accessed on 13 November 2020).

103. lf2653/Myrepository: Ros Driver for Continental ARS 408 Radar. Available online: https://github.com/lf2653/myrepository (accessed on 13 November 2020).

104. Smartmicro Automotive Radar UMRR-96 Type 153 I AutonomouStuff. Available online: https:/ /autonomoustuff.com/products / smartmicro-automotive-radar-umrr-96 (accessed on 20 February 2020).

105. Narula, L.; LaChapelle, D.M.; Murrian, M.J.; Wooten, J.M.; Humphreys, T.E.; Toldi, E.d.; Morvant, G.; Lacambre, J.-B. TEX-CUP: The University of Texas Challenge for Urban Positioning. In Proceedings of the 2020 IEEE/ION Position, Location and Navigation Symposium (PLANS), Portland, OR, USA, 20-23 April 2020.

106. Stanislas, L.; Thierry, P. Characterisation of the Delphi Electronically Scanning Radar for robotics applications. In Proceedings on the Australasian Conference on Robotics and Automation 2015; Li., H., Kim, J., Eds.; Australian Robotics and Automation Association: Sydney, Australia, 2015; pp. 1-10.

107. Automotive Radar Comparison-System Plus Consulting. Available online: https://www.systemplus.fr/wp-content/uploads/ 2018/10/SP18368-Automotive-Radar-Comparison-2018-Sample-2.pdf (accessed on 30 December 2020).

108. Aptiv SRR2 Rear and Side Detection System I AutonomouStuff. Available online: https://autonomoustuff.com/products/aptivsrr2 (accessed on 13 November 2020).

109. Aptiv ESR 2.5 | AutonomouStuff. Available online: https://autonomoustuff.com/products/aptiv-esr-2-5-24v (accessed on 13 November 2020).

110. Continental ARS 408-21 | AutonomouStuff. Available online: https://autonomoustuff.com/products/continental-ars-408-21 (accessed on 13 November 2020).

111. Xu, F.; Wang, H.; Hu, B.; Ren, M. Road Boundaries Detection based on Modified Occupancy Grid Map Using Millimeter-wave Radar. Mob. Netw. Appl. 2020, 25, 1496-1503. [CrossRef]

112. Weber, C.; von Eichel-Streiber, J.; Rodrigo-Comino, J.; Altenburg, J.; Udelhoven, T. Automotive Radar in a UAV to Assess Earth Surface Processes and Land Responses. Sensors 2020, 20, 4463. [CrossRef] 
113. Automotive Radar I Smartmicro. Available online: https:/ / www.smartmicro.com/automotive-radar (accessed on 13 June 2020).

114. Bruns, T.; Software Engineer-Smartmicro, Braunschweig, Germany; Yeong, D.J.; Institute of Technology, Tralee, Kerry, Ireland. Personal communication, 2020.

115. Parker, M. Chapter 19-Pulse Doppler Radar. In Digital Signal Processing 101: Everything You Need to Know to Get Started, 2nd ed.; Elsevier: Amsterdam, The Netherlands, 2017; pp. 241-251.

116. Lee, R.S.; Inside Sales Manager, AutonomouStuff-Hexagon, Stockholm, Sweden; Yeong, D.J.; Institute of Technology, Tralee, Kerry, Ireland. Personal communication, 2020.

117. Jain, A.; Zhang, L.; Jiang, L. High-Fidelity Sensor Calibration for Autonomous Vehicles. 2019. Available online: https://medium. com/lyftself-driving/high-fidelity-sensor-calibration-for-autonomous-vehicles-6af06eba4c26 (accessed on 13 October 2020).

118. Bouain, M.; Ali, K.M.A.; Berdjag, D.; Fakhfakh, N.; Atitallah, R.B. An Embedded Multi-Sensor Data Fusion Design for Vehicle Perception Tasks. J. Commun. 2018, 13, 8-14. [CrossRef]

119. Lesson 3: Sensor Calibration-A Necessary Evil-Module 5: Putting It together-An Autonomous Vehicle State Estimator I Coursera. Available online: https:/ / www.coursera.org/lecture/state-estimation-localization-self-driving-cars/lesson-3-sensorcalibration-a-necessary-evil-jPb2Y (accessed on 15 June 2020).

120. Tzafestas, S.G. Introduction to Mobile Robot Control, 1st ed.; Elsevier: Waltham, MA, USA, 2014; pp. 479-530.

121. Montag, A.; Technical Solutions Engineer-EMEA Velodyne Europe, Rüsselsheim, Germany; Yeong, D.J.; Institute of Technology, Tralee, Kerry, Ireland. Personal Communication, 2020.

122. Mirzaei, F.M. Extrinsic and Intrinsic Sensor Calibration. Ph.D. Thesis, University of Minnesota, Minneapolis, MN, USA, 2013.

123. Nouira, H.; Deschaud, J.E.; Goulette, F. Point Cloud Refinement with a Target-Free Intrinsic Calibration of a Mobile Multi-Beam LiDAR System. In Proceedings of the International Archives of the Photogrammetry, Remote Sensing and Spatial Information Sciences, Prague, Czech Republic, 12-19 July 2019; pp. 359-366.

124. De la Escalera, A.; Armingol, J.M. Automatic Chessboard Detection for Intrinsic and Extrinsic Camera Parameter Calibration. Sensors 2010, 10, 2027-2044. [CrossRef]

125. Jackman, B.; Sarraj, A.; Walsh, F. Self-Calibration of Fish-Eye Camera for Advanced Assistance Systems. In Proceedings of the ICCV 2018: 20th International Conference on Connected Vehicles, Zurich, Switzerland, 15-16 January 2018.

126. Liu, Z.; Wu, Q.; Wu, S.; Pan, X. Flexible and accurate camera calibration using grid spherical images. Opt. Express 2017, 25, 15269-15285. [CrossRef] [PubMed]

127. Xiao, Y.; Ruan, X.; Chai, J.; Zhang, X.; Zhu, X. Online IMU Self-Calibration for Visual-Inertial Systems. Sensors 2019, $19,1624$. [CrossRef] [PubMed]

128. Camera_Calibration-ROS Wiki. Available online: http://wiki.ros.org/camera_calibration (accessed on 23 July 2020).

129. Glennie, C.; Lichti, D.D. Static Calibration and Analysis of the Velodyne HDL-64E S2 for High Accuracy Mobile Scanning. Remote Sens. 2010, 2, 1610-1624. [CrossRef]

130. Lecture 1: The Pinhole Camera Model. Available online: http:/ / opilab.utb.edu.co/computer-vision/alllectures.pdf (accessed on 7 January 2021).

131. Pinhole Camera Model I HediVision. Available online: https://hedivision.github.io/Pinhole.html (accessed on 7 January 2021).

132. Burger, W.; Burge, M.J. 1.4 Image Acquisition. In Digital Image Processing-An Algorithmic Introduction Using Java, 2nd ed.; Gries, D., Schneider, F.B., Eds.; Springer: London, UK, 2016; pp. 4-11.

133. Burger, W. Zhang's Camera Calibration Algorithm: In-Depth Tutorial and Implementation; HGB16-05; University of Applied Sciences Upper Austria, School of Informatics, Communications and Media, Dept. of Digital Media: Hagenberg, Austria, 2016; pp. 1-6.

134. Camera Calibration and 3D Reconstruction-OpenCV 2.4.13.7 documentation. Available online: https://docs.opencv.org/2.4/ modules/calib3d/doc/camera_calibration_and_3d_reconstruction.html (accessed on 16 October 2020).

135. Camera Model: Intrinsic Parameters-Hoàng-Ân Lê. Available online: https://lhoangan.github.io/camera-params/ (accessed on 8 January 2021).

136. Hartley, R.; Zisserman, A. Multiple View Geometry in Computer Vision, 2nd ed.; Cambridge University Press: Cambridge, UK, 2004; pp. 1-19.

137. What is Camera Calibration? -MATLAB \& Simulink. Available online: https://www.mathworks.com/help/vision/ug/cameracalibration.html (accessed on 7 January 2021).

138. Dissecting the Camera Matrix, Part 3: The Intrinsic Matrix. Available online: http:/ / ksimek.github.io/2013/08/13/intrinsic/ (accessed on 7 January 2021).

139. Pedersen, M.; Bengtson, S.H.; Gade, R.; Madsen, N.; Moeslund, T.B. Camera Calibration for Underwater 3D Reconstruction Based on Ray Tracing Using Snell's Law. In Proceedings of the 2018 IEEE/CVF Conference on Computer Vision and Pattern Recognition Workshops (CVPRW), Salt Lake City, UT, USA, 18-22 June 2018.

140. Muhovič, J.; Perš, J. Correcting Decalibration of Stereo Cameras in Self-Driving Vehicles. Sensors 2020, 20, 3241. [CrossRef]

141. Zhang, Z. A flexible new technique for camera calibration. IEEE Trans. Pattern Anal. Mach. Intell. 2000, 22, 1330-1334. [CrossRef]

142. Wang, J.; Shi, F.; Zhang, J.; Liu, Y. A new calibration model for lens distortion. Pattern Recognit. 2008, 41, 607-615. [CrossRef]

143. Velas, M.; Spanel, M.; Materna, Z.; Herout, A. Calibration of RGB Camera with Velodyne LiDAR. J. WSCG 2014, $2014,135-144$.

144. Schöller, G.; Schnettler, M.; Krämmer, A.; Hinz, G.; Bakovic, M.; Güzet, M.; Knoll, A. Targetless Rotational Auto-Calibration of Radar and Camera for Intelligent Transportation Systems. arXiv 2019, arXiv:1904.08743. 
145. An, P.; Ma, T.; Yu, K.; Fang, B.; Zhang, J.; Fu, W.; Ma, J. Geometric calibration for LiDAR-camera system fusing 3D-2D and 3D-3D point correspondences. Opt. Express 2020, 28, 2122-2141. [CrossRef]

146. Domhof, J.; Kooij, J.F.P.; Gavrila, D.M. An Extrinsic Calibration Tool for Radar, Camera and Lidar. In Proceedings of the 2019 International Conference on Robotics and Automation (ICRA), Montreal, QC, Canada, 20-24 May 2019.

147. tudelft-iv/multi_sensor_calibration. Available online: https://github.com/tudelft-iv/multi_sensor_calibration (accessed on 16 July 2020).

148. Peršić, J.; Marković, I.; Petrović, I. Extrinsic 6DoF calibration of a radar-LiDAR-camera system enhanced by radar cross section estimates evaluation. Rob. Auton. Syst. 2019, 114, 217-230. [CrossRef]

149. Peršić, J.; Marković, I.; Petrović, I. Extrinsic 6DoF calibration of 3D LiDAR and radar. In Proceedings of the 2017 European Conference on Mobile Robots (ECMR), Paris, France, 6-8 September 2017.

150. Mishra, S.; Pandey, G.; Saripalli, S. Extrinsic Calibration of a 3D-LIDAR and a Camera. arXiv 2020, arXiv:2003.01213v2.

151. Jeong, J.; Cho, L.Y.; Kim, A. Road is Enough! Extrinsic Calibration of Non-overlapping Stereo Camera and LiDAR using Road Information. arXiv 2019, arXiv:1902.10586v2. [CrossRef]

152. Huang, J.K.; Grizzle, J.W. Improvements to Target-Based 3D LiDAR to Camera Calibration. IEEE Access 2020, 8, 134101-134110. [CrossRef]

153. UMich-BipedLab/extrinsic_lidar_camera_calibration: This is a package for extrinsic calibration between a 3D LiDAR and a camera, described in paper: Improvements to Target-Based 3D LiDAR to Camera Calibration. This package is used for Cassie Blue's 3D LiDAR semantic mapping and automation. Available online: https:/ / github.com/UMich-BipedLab/extrinsic_lidar_ camera_calibration (accessed on 15 January 2021).

154. Beltrán, J.; Guindel, C.; García, F. Automatic Extrinsic Calibration Method for LiDAR and Camera Sensor Setups. arXiv 2021, arXiv:2101.04431.

155. velo2cam_calibration-ROS Wiki. Available online: http://wiki.ros.org/velo2cam_calibration (accessed on 15 January 2021).

156. Dhall, A.; Chelani, K.; Radhakrishnan, V.; Krishna, K.M. LiDAR-Camera Calibration using 3D-3D Point correspondences. arXiv 2017, arXiv:1705.09785.

157. Ankitdhall/Lidar_Camera_Calibration: ROS Package to Find a Rigid-Body Transformation between a LiDAR and a Camera for "LiDAR-Camera Calibration Using 3D-3D Point Correspondences". Available online: https://github.com/ankitdhall/lidar_ camera_calibration\#usage (accessed on 16 July 2020).

158. But_Calibration_Camera_Velodyne-ROS Wiki. Available online: http:/ / wiki.ros.org/but_calibration_camera_velodyne (accessed on 16 July 2020).

159. Yin, L.; Luo, B.; Wang, W.; Yu, H.; Wang, C.; Li, C. CoMask: Corresponding Mask-Based End-to-End Extrinsic Calibration of the Camera and LiDAR. Remote Sens. 2020, 12, 1925. [CrossRef]

160. Autoware Camera-LiDAR Calibration Package-Autoware 1.9.0 Documentation. Available online: https:/ / autoware.readthedocs. io/en/feature-documentation_rtd/DevelopersGuide/PackagesAPI/sensing/autoware_camera_lidar_calibrator.html (accessed on 15 January 2021).

161. Guindel, C.; Beltrán, J.; Martín, D.; García, F. Automatic extrinsic calibration for lidar-stereo vehicle sensor setups. In Proceedings of the 2017 IEEE 20th International Conference on Intelligent Transportation Systems (ITSC), Yokohama, Japan, 16-19 October 2017; pp. 674-679.

162. Products I Velodyne Lidar. Available online: https://velodynelidar.com/products/ (accessed on 18 January 2021).

163. Sensor_Msgs_ROS Wiki. Available online: http:/ / wiki.ros.org/sensor_msgs (accessed on 18 January 2021).

164. Message_Filters_ROS Wiki. Available online: http:/ / wiki.ros.org/message_filters (accessed on 17 July 2020).

165. Chapter 9: Time Synchronization. Available online: https://www3.nd.edu/ \{\}cpoellab/teaching/cse40815/Chapter9.pdf (accessed on 22 March 2020).

166. Kelly, J.; Sukhatme, G.S. A General Framework for Temporal Calibration of Multiple Proprioceptive and Exteroceptive Sensors. In Experiment Robotics; Khatib, O., Kumar, V., Sukhatme, G., Eds.; Springer: Berlin/Heidelberg, Germany, 2014; Volume 79, pp. 195-209.

167. Abdelmohsen, Y.K. Camera-LIDAR Detection Fusion. Bachelor's Thesis, German University in Cairo, New Cairo City, Egypt, 2020.

168. Olson, E. A passive solution to the sensor synchronization problem. In Proceedings of the 2010 IEEE/RSJ International Conference on Intelligent Robots and Systems, Taipei, Taiwan, 18-22 October 2010.

169. Peršić, J.; Petrović, L.; Marković, I.; Petrović, I. Spatio-Temporal Multisensor Calibration Based on Gaussian Processes Moving Object Tracking. arXiv 2019, arXiv:1904.04187.

170. Unizg-fer-Lamor / Calirad-Bitbucket. Available online: https://bitbucket.org/unizg-fer-lamor/calirad/src/master/ (accessed on 15 May 2020).

171. Spatiotemporal Multisensor Calibration via Gaussian Process Moving Target Tracking-YouTube. Available online: https: / / www.youtube.com/watch?v=vqTR6zMIKJs\&ab_channel=LAMOR (accessed on 15 May 2020).

172. Peršić, J.; University of Zagreb, Zagreb, Croatia; Yeong, D.J.; Munster Technological University, Tralee, Ireland. Personal Communication, 2020. 
173. Lee, C.-L.; Hsueh, Y.-H.; Wang, C.-C.; Lin, W.-C. Extrinsic and Temporal Calibration of Automotive Radar and 3D LiDAR. In Proceedings of the 2020 IEEE/RSJ International Conference on Intelligent Robots and Systems (IROS), Las Vegas, NV, USA, 25-29 October 2020.

174. Rangesh, A.; Yuen, K.; Satzoda, R.K.; Rajaram, R.N.; Gunaratne, P.; Trivedi, M.M. A Multimodal, Full-Surround Vehicular Testbed for Naturalistic Studies and Benchmarking: Design, Calibration and Deployment. arXiv 2019, arXiv:1709.07502v4.

175. Lundquist, C. Sensor Fusion for Automotive Applications; Linköping University: Linköping, Sweden, 2011.

176. Pollach, M.; Schiegg, F.; Knoll, A. Low Latency and Low-Level Sensor Fusion for Automotive Use-Cases. In Proceedings of the 2020 IEEE International Conference on Robotics and Automation (ICRA), Paris, France, 31 May-31 August 2020.

177. Gu, S.; Zhang, Y.; Yang, J.; Alvarez, J.M.; Kong, H. Two-View Fusion based Convolutional Neural Network for Urban Road Detection. In Proceedings of the IEEE/RSJ International Conference on Intelligent Robots and Systems (IROS), Macau, China, 3-8 November 2019.

178. Nobis, F.; Geisslinger, M.; Weber, M.; Betz, J.; Lienkamp, M. A Deep Learning-based Radar and Camera Sensor Fusion Architecture for Object Detection. In Proceedings of the 2019 Sensor Data Fusion: Trends, Solutions, Applications (SDF), Bonn, Germany, 15-17 October 2019.

179. Self-Driving Made Real-NAVYA. Available online: https:/ / navya.tech/fr (accessed on 25 January 2021).

180. Banerjee, K.; Notz, D.; Windelen, J.; Gavarraju, S.; He, M. Online Camera LiDAR Fusion and Object Detection on Hybrid Data for Autonomous Driving. In Proceedings of the 2018 IEEE Intelligent Vehicles Symposium (IV), Changshu, China, 26-30 June 2018

181. Yoo, J.H.; Kim, Y.; Kim, J.; Choi, J.W. 3D-CVF: Generating Joint Camera and LiDAR Features Using Cross-View Spatial Feature Fursion for 3D Object Detection. arXiv 2020, arXiv:2004.12636v2.

182. Li, Y.; Jha, D.K.; Ray, A.; Wettergren, T.A. Feature level sensor fusion for target detection in dynamic environments. In Proceedings of the 2015 American Control Conference (ACC), Chicago, IL, USA, 1-3 July 2015.

183. Visteon I Current Sensor Data Fusion Architectures: Visteon's Approach. Available online: https://www.visteon.com/currentsensor-data-fusion-architectures-visteons-approach/ (accessed on 28 January 2021).

184. Brena, R.F.; Aguileta, A.A.; Trejo, L.A.; Molino-Minero-Re, E.; Mayora, O. Choosing the Best Sensor Fusion Method: A MachineLearning Approach. Sensors 2020, 20, 2350. [CrossRef]

185. Rosique, F.; Navarro, P.J.; Fernández, C.; Padilla, A. A Systematic Review of Perception System and Simulators for Autonomous Vehicles Research. Sensors 2019, 19, 648. [CrossRef] [PubMed]

186. Ali, M.A.H.; Mailah, M.; Jabbar, W.A.; Moiduddin, K.; Ameen, W.; Alkhalefah, H. Autonomous Road Roundabout Detection and Navigation System for Smart Vehicles and Cities Using Laser Simulator-Fuzzy Logic Algorithms and Sensor Fusion. Sensors 2020, 20, 3694. [CrossRef]

187. Kim, J.; Kim, J.; Cho, J. An advanced object classification strategy using YOLO through camera and LiDAR sensor fusion. In Proceedings of the 2019 13th International Conference on Signal Processing and Communication Systems (ICSPCS), Gold Coast, Australia, 16-18 December 2019.

188. Redmon, J.; Divvala, S.; Girshick, R.; Farhadi, A. You Only Look Once: Unified, Real-Time Object Detection. arXiv 2016, arXiv:1506.02640v5.

189. Bochkovskiy, A.; Wang, C.-Y.; Liao, M.H. YOLOv4: Optimal Speed and Accuracy of Object Detection. arXiv 2020, arXiv:2004.10934.

190. Lee, K.W.; Yoon, H.S.; Song, J.M.; Park, K.R. Convolutional Neural Network-Based Classification of Driver's Emotion during Aggressive and Smooth Driving Using Multi-Modal Camera Sensors. Sensors 2018, 18, 957. [CrossRef] [PubMed]

191. Sindagi, V.A.; Zhou, Y.; Tuzel, O. MVX-Net: Multimodal VoxelNet for 3D Object Detection. arXiv 2019, arXiv:1904.01649.

192. Zhou, Y.; Tuzel, O. VoxelNet: End-to-End Learning for Point Cloud Based 3D Object Detection. arXiv 2017, arXiv:1711.06396.

193. Xu, D.; Anguelov, D.; Jain, A. PointFusion: Deep Sensor Fusion for 3D Bounding Box Estimation. arXiv 2018, arXiv:1711.10871v2.

194. Qi, C.R.; Su, H.; Mo, K.; Guibas, L.J. PointNet: Deep Learning on Point Sets for 3D Classification and Segmentation. arXiv 2017, arXiv:1612.00593v2.

195. He, K.; Zhang, X.; Ren, S.; Sun, J. Deep Residual Learning for Image Recognition. arXiv 2015, arXiv:1512.03385.

196. Liu, W.; Anguelov, D.; Erhan, D.; Szegedy, C.; Reed, S.; Fu, C.-Y.; Berg, A.C. SSD: Single Shot MultiBox Detector. arXiv 2015, arXiv:1512.02325.

197. Zhou, X.; Wang, D.; Krähenbühl, P. Objects as Points. arXiv 2019, arXiv:1904.07850v2.

198. O'Mahony, N.; Campbell, S.; Carvalho, A.; Harapanahalli, S.; Velasco-Hernandez, G.; Krpalkova, L.; Riordan, D.; Walsh, J. Deep Learning vs. Traditional Computer Vision. arXiv 2019, arXiv:1910.13796.

199. Bhanushali, D.R. Multi-Sensor Fusion for 3D Object Detection. Master's Thesis, Rochester Institute of Technology, New York, NY, USA, 2020.

200. Shi, W.; Bao, S.; Tan, D. FFESSD: An Accurate and Efficient Single-Shot Detector for Target Detection. Appl. Sci. 2019, 9, 4276. [CrossRef]

201. Nabati, R.; Qi, H. CenterFusion: Center-based Radar and Camera Fusion for 3D Object Detection. arXiv 2020, arXiv:2011.04841v1.

202. Roth, M.; Jargot, D.; Gavrila, D.M. Deep End-to-end 3D Person Detection from Camera and Lidar. In Proceedings of the 2019 IEEE Intelligent Transportation Systems Conference (ITSC), Auckland, New Zealand, 27-30 October 2019.

203. Zhou, Y.; Sun, P.; Zhang, Y.; Anguelov, D.; Gao, J.; Ouyang, T.; Guo, J.; Ngiam, J.; Vasudevan, V. End-to-End Mult-View Fusion for 3D Object Detection in LiDAR Point Clouds. arXiv 2019, arXiv:1910.06528v2. 
204. Elfring, J.; Appeldoorn, R.; van den Dries, S.; Kwakkernaat, M. Effective World Modeling: Multisensor Data Fusion Methodology for Automated Driving. Sensors 2016, 16, 1668. [CrossRef]

205. Floudas, N.; Polychronopoulos, A.; Aycard, O.; Burlet, J.; Ahrholdt, M. High Level Sensor Data Fusion Approaches for Object Recognition in Road Environment. In Proceedings of the 2007 IEEE Intelligent Vehicles Symposium, Istanbul, Turkey, 13-15 June 2007.

206. Kim, S.; Song, W.; Kim, S. Double Weight-Based SAR and Infrared Sensor Fusion for Automatic Ground Target Recognition with Deep Learning. Remote Sens. 2018, 10, 72. [CrossRef]

207. Miller, R. Rolling Zettabytes: Quantifying the Data Impact of Connected Cars. Available online: https://datacenterfrontier.com/ rolling-zettabytes-quantifying-the-data-impact-of-connected-cars/ (accessed on 1 February 2021).

208. Liu, S.; Tang, J.; Zhang, Z.; Gaudiot, J.-L. CAAD: Computer Architecture for Autonomous Driving. arXiv 2017, arXiv:1702.01894.

209. Knight, W. An Ambitious Plan to Build a Self-Driving Borg. Available online: https://www.technologyreview.com/2016/10/10 /157091/an-ambitious-plan-to-build-a-self-driving-borg/ (accessed on 1 February 2021).

210. Wiggers, K. Roboflow: Popular autonomous vehicle data set contains critical flaws I VentureBeat. Available online: https://venturebeat.com/2020/02/14/report-popular-autonomous-vehicle-data-set-contains-critical-flaws/ (accessed on 1 February 2021).

211. Ren, K.; Zheng, T.; Qin, Z.; Liu, X. Adversarial Attacks and Defenses in Deep Learning. Engineering 2020, 6, 346-360. [CrossRef]

212. Ma, X.; Niu, Y.; Gu, L.; Wang, Y.; Zhao, Y.; Bailey, J.; Lu, F. Understanding Adversarial Attacks on Deep Learning Based Medical Image Analysis Systems. arXiv 2020, arXiv:1907.10456v2.

213. Yurtsever, E.; Lambert, J.; Carballo, A.; Takeda, K. A Survey of Autonomous Driving: Common Practices and Emerging Technologies. IEEE Access 2020, 8, 58443-58469. [CrossRef]

214. Rawat, P. Environment Perception for Autonomous Driving: A 1/10 Scale Implementation of Low-Level Sensor Fusion Using Occupancy Grid Mapping. Master's Thesis, KTH Royal Institute of Technology, Stockholm, Sweden, March 2019.

215. Kiran, B.R.; Sobh, I.; Talpaert, V.; Mannion, P.; Al Sallab, A.A.; Yogamani, S.; Pérez, P. Deep Reinforcement Learning for Autonomous Driving: A Survey. arXiv 2021, arXiv:2002.00444v2. 